TRANSACTIONS OF THE

AMERICAN MATHEMATICAL SOCIETY

Volume 349, Number 7, July 1997, Pages 2619-2661

S 0002-9947(97)01718-2

\title{
THE ITERATION FORMULA \\ OF THE MASLOV-TYPE INDEX THEORY WITH APPLICATIONS TO NONLINEAR HAMILTONIAN SYSTEMS
}

\author{
DI DONG AND YIMING LONG
}

\begin{abstract}
In this paper, the iteration formula of the Maslov-type index theory for linear Hamiltonian systems with continuous, periodic, and symmetric coefficients is established. This formula yields a new method to determine the minimality of the period for solutions of nonlinear autonomous Hamiltonian systems via their Maslov-type indices. Applications of this formula give new results on the existence of periodic solutions with prescribed minimal period for such systems, and unify known results under various convexity conditions.
\end{abstract}

\section{INTRODUCTION AND MAIN RESULTS}

In this paper, we consider the existence of nonconstant periodic solutions with prescribed minimal period for the following autonomous nonlinear Hamiltonian systems:

$$
\dot{x}=J H^{\prime}(x), \quad \forall x \in \mathbf{R}^{2 n} .
$$

Here $n$ is a positive integer, $H: \mathbf{R}^{2 n} \rightarrow \mathbf{R}$ is a smooth function, $H^{\prime}$ denotes its gradient, and $J=\left(\begin{array}{cc}0 & -I \\ I & 0\end{array}\right)$, where $I$ is the identity matrix on $\mathbf{R}^{n}$.

In his pioneer work [Ra1] of 1978, Rabinowitz proved the existence of nonconstant prescribed periodic solutions of (1.1). Because a $\frac{T}{k}$-periodic function is also a $T$ periodic function for every $k \in \mathbf{N}$, Rabinowitz conjectured that (1.1) possesses a nonconstant solution with any prescribed minimal period under suitable conditions. Since then, many contributions on this minimal period problem have been made by many mathematicians. Among all these results, two kinds of methods are used to determine the minimality of the period of a solution. The first method depends on a priori estimates for the solutions, and is used by many authors (cf. [AM], [CE1, CE2], [De], [Ek3], [GM1, GM2], [Lo8]). The second method depends on the dual action principle of convex Hamiltonian systems, the iteration inequality of MorseEkeland index theory, Bott's formula, and Hofer's topological characterization of mountain-pass points. This method was first introduced by Ekeland and Hofer in their celebrated paper $[\mathrm{EH}]$, and has been used by many other authors to various

Received by the editors May 3, 1994.

1991 Mathematics Subject Classification. Primary 58F05, 58E05, 34C25; Secondary 15A18, 15A21.

Key words and phrases. Normal form of a symplectic matrix, perturbation of eigenvalues, symplectic path, Maslov-type index, iteration formula, minimal period, Hamiltonian systems.

The second author was partially supported by YTF of Edu. Comm., NNSF of China, and Qiu Shi Sci. and Tech. Foundations. 
convex Hamiltonian systems (cf. [AC], [Ek3], [GM3, GM4, GM5], [Zh]). Note that recently this method has been partially extended to second order Hamiltonian systems without convexity conditions by [Lo4, Lo5, Lo6].

The present paper uses neither of these two methods. Our approach to this prescribed minimal period solution problem depends on a new tool: the iteration formula of the Maslov-type index theory for linear Hamiltonian systems with periodic continuous and symmetric coefficients. The main part of this paper is devoted to the establishment of this formula. Then we apply it to nonlinear problems, and show that the minimality of the period of a solution can be determined from only the information carried by its Maslov-type indices.

Consider the linear Hamiltonian systems

$$
\dot{y}=J B(t) y, \quad x \in \mathbf{R}^{2 n},
$$

where for $T>0$ we have $B \in C\left(S_{T}, \mathcal{L}_{s}\left(\mathbf{R}^{2 n}\right)\right)$ and $S_{T}=\mathbf{R} /(T \mathbf{Z})$, while $\mathcal{L}_{s}\left(\mathbf{R}^{2 n}\right)$ is the set of all symmetric real $2 n \times 2 n$ matrices. Denote by $\gamma(t)$ for $0 \leq t \leq T$ the fundamental solution of (1.2). The Maslov-type index theory for such linear systems was first established by Conley and Zehnder (cf. [CZ]) in 1983 when $n \geq 2$ and (1.2) is nondegenerate, i.e. $\operatorname{det}(\gamma(T)-I) \neq 0$. This index theory was extended by Long and Zehnder (cf. [LZ]) in 1988 to nondegenerate linear systems with $n=1$, and by Long (cf. [Lo1, Lo2]) in 1990 to degenerate linear systems. For each system (1.2), its Maslov-type index is a pair of integers, $\left(i_{T}, \nu_{T}\right)=\left(i_{T}(B), \nu_{T}(B)\right) \in \mathbf{Z} \times\{0, \ldots, 2 n\}$, where $i_{T}$ is the index part and $\nu_{T} \equiv \operatorname{dim} \operatorname{ker}(\gamma(T)-I)$ is the nullity. If $x$ is a $T$ periodic solution of a Hamiltonian system

$$
\dot{x}(t)=J H^{\prime}(t, x(t)), \quad x \in \mathbf{R}^{2 n},
$$

then $\left(i_{T}(B), \nu_{T}(B)\right)$ with $B(t)=H^{\prime \prime}(t, x(t))$ is defined to be the Maslov-type index of the solution $x$, and is denoted by $\left(i_{T}(x), \nu_{T}(x)\right)$. Note that every nonconstant $T$-periodic solution $x$ of the autonomous system (1.1) is always degenerate, i.e. we always have $\nu_{T}(x) \geq 1$. Note also that the Morse indices of the functional corresponding to the system (1.3) are always infinite. The Maslov-type index theory gives a finite representation for the corresponding Morse index theory.

Our iteration formula of the Maslov-type index theory is established in Theorems 4.1 and 8.3. This formula gives a relationship between $\left(i_{k T}, \nu_{k T}\right)$ and $\left(i_{T}, \nu_{T}\right)$. In Theorem 4.1, it is proved that if $\nu_{k T}=0$, then $\nu_{T}=0$ and

$$
i_{k T}=k\left(i_{T}-\mu\right)+\sum_{j=1}^{\mu} 2 t_{j}-\mu,
$$

and in case $\mu=n$, the integers $\mu, i_{T}$, and $i_{k T}$ possess the same parity. This formula depends on normal forms of the matrix $\gamma(T)$. In Theorem 8.3, the formula (1.4) is extended to the case of $\nu_{k T}>0$, and $i_{k T}$ is estimated by indices of nearby nondegenerate paths. The proof of this formula depends on a complete understanding of symplectic matrices under iteration, and careful perturbation arguments on the eigenvalues of symplectic matrices which are $k$ th roots of unity. The perturbation of the eigenvalue 1 of $\gamma(T)$ has been studied in [Lo1, Lo2]. In this paper the perturbation of -1 and other $k$ th roots of unity as eigenvalues of $\gamma(T)$ are studied in detail. Then these results are applied to the study of paths in the symplectic group to get the expected iteration formula. Some of the ideas in these proofs are contained in [CZ], [LZ], and [Lo1, Lo2]. 
A direct consequence of this iteration formula is that for the linear system (1.2), if $n+1-\nu_{k T} \leq i_{k T} \leq n+1, \nu_{T} \geq 1$, and $n=1$, then $k=1$ (cf. Theorem 11.1). Thus if $H \in C^{2}\left(\mathbf{R}^{2}, \mathbf{R}\right)$, and $x$ is a nonconstant $T$-periodic solution of the nonlinear autonomous system (1.1) satisfying $2-\nu_{T}(x) \leq i_{T}(x) \leq 2$, then $x$ must possess minimal period $T$ (cf. Theorem 11.2). Consequently the existence of periodic solutions of (1.1) obtained via the saddle point theorem implies the minimality of the period (cf. Theorem 11.5) with no requirements on the second order derivatives of the Hamiltonian functions.

Unfortunately, this argument for systems on $\mathbf{R}^{2}$ fails for higher-dimensional cases (cf. Example 11.6). To further our study for the general-dimensional case, a natural additional condition is $i_{T} \geq n$, which includes the convex Hamiltonians on $\mathbf{R}^{2 n}$ as a special case. A typical consequence of the iteration formula is that for the linear system (1.2), if $i_{k T} \leq n+1, i_{T} \geq n$, and $\nu_{T} \geq 1$, then $k=1$ (cf. Theorem 9.1 and Corollary 9.2). In a certain sense, this result actually unifies all the results known so far on this minimal period problem for periodic solutions of Hamiltonian systems under various convexity conditions. Such a theorem can be viewed as a generalization of the following observation on the simplest convex linear Hamiltonian system (1.2) with $n=1, T=2 \pi$, and $B(t)=I$. In this case,

$$
\gamma(t)=\left(\begin{array}{cc}
\cos t & -\sin t \\
\sin t & \cos t
\end{array}\right)
$$

and $i_{k T}=2 k-1$ and $\nu_{k T}=2$ for any integer $k$. Thus if the increment of the Maslov-type index is less than 2, there is actually no iteration.

For nonlinear Hamiltonian systems, suppose that $H \in C^{2}\left(\mathbf{R}^{2 n}, \mathbf{R}\right)$ and $x$ is a nonconstant $T$-periodic solution of (1.1), and denote the minimal period of $x$ by $\frac{T}{k}$ for some integer $k \geq 1$. If the Maslov-type indices of $x$ satisfy $i_{T}(x) \leq$ $n+1$ and $i_{T / k}(x) \geq n$, then our above results imply $k=1$, i.e. $x$ has minimal period $T$ (cf. Theorem 9.3). Here for example, the requirement $i_{T}(x) \leq n+1$ is satisfied by solutions found via the saddle point theorem of Rabinowitz, and the requirement $i_{T / k}(x) \geq n$ follows from a convexity condition on $H$ along the orbit of $x$. Thus, especially, we can further apply our results to convex Hamiltonian systems, and obtain some strict generalizations (cf. Theorem 10.1 and Corollary 10.5) of a theorem of Ekeland and Hofer (Theorem IV.4 of [EH]).

Note that, in contrast to the second method mentioned above, our study of the structure of symplectic matrices, perturbations of their eigenvalues, and the iteration formula of the Maslov-type index theory already yields enough information in terms of Maslov-type indices for the determination of the minimal period of a given periodic solution, and it is not necessary to invoke Bott's formula, or Hofer's characterization of mountain-pass points. Note also that our iteration formula of the Maslov-type index theory together with its proof is very different from those iteration formulae and their proofs of various index theories in [Bo], [CD], [Ek1, Ek2, Ek3], [K1], and [Lo4, Lo5, Lo6]. On the other hand, our method and results in this paper are more related to the pioneer works of Gel'fand and Lidskiı [GL] and Moser $[\mathrm{Mo}]$ on linear Hamiltonian systems.

In this paper, we denote by $\mathbf{N}, \mathbf{Z}, \mathbf{R}$, and $\mathbf{C}$ the natural, integral, real, and complex numbers respectively, and by $\mathbf{U}$ the unit circle in $\mathbf{C}$. Denote by $|a|$ and $a \cdot b$ the usual norm and inner product in $\mathbf{R}^{2 n}$. This paper is organized as follows:

1. Introduction and main results.

2. The Maslov-type index theory and its homotopy invariance. 
3. Normal forms of nonsingular symplectic matrices.

4. The iteration formula for nondegenerate linear Hamiltonian systems.

5. Perturbations of eigenvalues away from 1.

6. Perturbations of eigenvalues away from -1 .

7. Perturbations of eigenvalues away from roots of unity $e^{ \pm \theta \sqrt{-1}} \notin \mathbf{R}$.

8. The iteration formula for degenerate linear Hamiltonian systems.

9. Controlling the minimal period via Maslov-type indices.

10. Applications to autonomous nonlinear Hamiltonian systems.

11. Applications to Hamiltonian systems on $\mathbf{R}^{2}$.

\section{The Maslov-TyPe indeX TheORY AND ITS HOMOTOPY INVARIANCE}

In this section, first, we briefly recall the definition of Maslov-type index theory for periodic solutions of Hamiltonian systems given by [CZ], [LZ], and [Lo1, Lo2]. For a complete description we refer to [Lo7]. Then we prove the homotopy invariance of this index theory.

Let $\operatorname{Sp}(2 n)=\left\{M \in \mathcal{L}\left(\mathbf{R}^{2 n}\right) \mid M^{T} J M=J\right\}, \operatorname{Sp}(2 n)^{*}=\{M \in \operatorname{Sp}(2 n) \mid \operatorname{det}(M-I)$ $\neq 0\}$, and $\operatorname{Sp}(2 n)^{0}=\operatorname{Sp}(2 n) \backslash \operatorname{Sp}(2 n)^{*}$. Note that $\operatorname{Sp}(2 n)^{*}$ consists of two pathconnected components $\operatorname{Sp}(2 n)^{ \pm}=\{M \in \operatorname{Sp}(2 n) \mid \pm \operatorname{det}(M-I)<0\}$.

Fix $T>0$, and let $P=\{\gamma \in C([0, T], \operatorname{Sp}(2 n)) \mid \gamma(0)=I\}$ and $P^{*}=\{\gamma \in$ $\left.P \mid \gamma(T) \in \operatorname{Sp}(2 n)^{*}\right\}$. Define $\mathcal{P}$ to be the set of all paths $\gamma \in C^{1}([0, T], \operatorname{Sp}(2 n))$ such that $\gamma(0)=I$ and $B(\cdot) \equiv-J \dot{\gamma}(\cdot) \gamma^{-1}(\cdot) \in C\left(S_{T}, \mathcal{L}_{S}\left(\mathbf{R}^{2 n}\right)\right)$. Here $B(t)$ is symmetric, since $\gamma$ is a symplectic path. Note that $\gamma \in \mathcal{P}$ is the fundamental solution of the following linear Hamiltonian system with $B$ defined above:

$$
\dot{y}=J B(t) y \text {. }
$$

Definition 2.1 (cf. [Lo1]). For every $\gamma \in P$, we define $\nu_{T}(\gamma)=\operatorname{dim} \operatorname{ker}(\gamma(T)-I)$.

Definition 2.2 (cf. [Lo1]). Two paths $\gamma_{0}$ and $\gamma_{1} \in P$ are homotopic to each other, and we write $\gamma_{0} \sim \gamma_{1}$, if there is a map $\delta \in C([0,1] \times[0, T], \operatorname{Sp}(2 n))$ such that $\delta(0, t)=\gamma_{0}(t), \delta(1, t)=\gamma_{1}(t), \delta(s, 0)=I$, and $\nu_{T}(\delta(s, T))$ is constant for $0 \leq s \leq 1$.

As is well known, every $M \in \operatorname{Sp}(2 n)$ has its unique polar decomposition $M=$ $A U$, where $A=\left(M M^{T}\right)^{1 / 2}$ is symmetric, positive definite and symplectic, while $U$ is orthogonal and symplectic. Therefore $U$ has form

$$
U=\left(\begin{array}{cc}
u_{1} & -u_{2} \\
u_{2} & u_{1}
\end{array}\right)
$$

where $u=u_{1}+\sqrt{-1} u_{2} \in \mathcal{L}\left(\mathbf{C}^{n}\right)$ is a unitary matrix. So to every path $\gamma:[0, T] \rightarrow$ $\operatorname{Sp}(2 n)$ we can associate a path $u(t)$ in the unitary group on $\mathbf{C}^{n}$. If $\Delta(t)$ is any continuous real function satisfying $\operatorname{det} u(t)=\exp (\sqrt{-1} \Delta(t))$, the difference $\Delta(T)-$ $\Delta(0)$ depends only on $\gamma$ but not on the choice of the function $\Delta(t)$. Therefore we may define

$$
\Delta_{T}(\gamma)=\Delta(T)-\Delta(0) .
$$

Lemma 2.3 (cf. [CZ], [LZ]). If $\gamma_{1}$ and $\gamma_{2} \in P^{*}$ and possess common end points, then $\gamma_{1} \sim \gamma_{2}$ if and only if $\Delta_{T}\left(\gamma_{1}\right)=\Delta_{T}\left(\gamma_{2}\right)$, and this homotopy can be chosen to keep their end points fixed. 
For any $\gamma \in P^{*}$, we can connect $\gamma(T)$ to $-I$ or $\operatorname{diag}\left(\frac{1}{2},-I, 2,-I\right)$ by a path $\beta$ in $\operatorname{Sp}(2 n) *$ to get a product path $\beta * \gamma$, where

$$
\beta * \gamma(t)= \begin{cases}\gamma(2 t), & 0 \leq t \leq T / 2 \\ \beta(2 t-T), & T / 2<t \leq T\end{cases}
$$

Then $k \equiv \Delta_{T}(\beta * \gamma) / \pi \in \mathbf{Z}$ and is independent of the choice of the path $\beta$. In this case we write $\gamma \in P_{k}$. These $P_{k}$ 's give a homotopy classification of $P^{*}$.

Definition 2.4 (cf. [CZ], [LZ]). If $\gamma \in P_{k}$, we define $i_{T}(\gamma)=k$.

For every integer $m, 1 \leq m \leq n$, and $\theta \in \mathbf{R}$, a $2 n \times 2 n$ rotation matrix $R_{m}(\theta)=$ $\left(r_{i, j}\right)$ is defined in [Lo1, Lo2] by

$$
\left\{\begin{array}{l}
r_{m, m}=r_{n+m, n+m}=\cos \theta \\
r_{n+m, m}=-r_{m, n+m}=\sin \theta \\
r_{i, i}=1, \quad \text { if } i \neq m, n+m \\
r_{i, j}=0, \quad \text { otherwise. }
\end{array}\right.
$$

Let $\mathcal{P}^{0}=\left\{\gamma \in \mathcal{P} \mid \gamma(T) \in \operatorname{Sp}(2 n)^{0}\right\}$. Fix $\gamma \in \mathcal{P}^{0}$. For $t_{0} \in(0, T)$, let $\rho \in$ $C^{2}([0, T],[0,1])$ be such that $\rho(t)=0$ for $0 \leq t \leq t_{0}, \dot{\rho}(t) \geq 0$ for $0 \leq t \leq T$, $\rho(T)=1$, and $\dot{\rho}(T)=0$. In [Lo1, Lo2, Lo9] it is proved that there exist $Q \in$ $\operatorname{Sp}(2 n)$, an integer $q, 1 \leq q \leq n$, a strictly increasing subsequence $\left\{m_{1}, \ldots, m_{q}\right\}$ of $\{1, \ldots, n\}, t_{0} \in(0, T)$ close to $T$, and $\theta_{0} \in(0, \pi / 8 n)$ small enough that for any $(s, t) \in[-1,1] \times[0, T]$ the paths

$$
\gamma_{s}(t)=\gamma(t) Q^{-1} R_{m_{1}}\left(s \rho(t) \theta_{0}\right) \cdots R_{m_{q}}\left(s \rho(t) \theta_{0}\right) Q
$$

satisfy $\gamma_{0}=\gamma, \gamma_{s}(t)=\gamma(t)$ for $0 \leq t \leq t_{0}, \gamma_{s}$ converges to $\gamma$ in $C^{1}$ as $s \rightarrow 0$, and

$$
\begin{gathered}
\nu_{T}\left(\gamma_{s}\right)=0, \quad \text { if } s \neq 0, \\
i_{T}\left(\gamma_{s}\right)=i_{T}\left(\gamma_{s^{\prime}}\right), i\left(\gamma_{-s}\right)=i\left(\gamma_{-s^{\prime}}\right), \quad \forall s, s^{\prime} \in(0,1], \\
i_{T}\left(\gamma_{s}\right)-i_{T}\left(\gamma_{-s}\right)=\nu_{T}(\gamma), \quad \forall s \in(0,1] .
\end{gathered}
$$

Definition 2.5 (cf. [Lo1]). Define $i_{T}(\gamma)=i_{T}\left(\gamma_{-s}\right)$ for $s \in(0,1]$.

Definition 2.6 (cf. [Lo1]). Definitions 2.1, 2.4 and 2.5 assign a pair of integers $\left(i_{T}(\gamma), \nu_{T}(\gamma)\right) \in \mathbf{Z} \times\{0, \ldots, 2 n\}$ to every path $\gamma \in \mathcal{P}$. This pair of integers is called the Maslov-type index of $\gamma$, and of the corresponding $B(t)$ in (2.1).

Let $E=L^{2}\left(0, T ; \mathbf{R}^{2 n}\right)$. Define $A x=-J \dot{x}$, with $\operatorname{dom} A=\left\{x \in W^{1,2}\left(0, T ; \mathbf{R}^{2 n}\right) \mid\right.$ $x(0)=x(T)\}$. Then $A=A^{*}$ and $\sigma(A)=\frac{2 \pi}{T} \mathbf{Z}$. Given $H \in C^{2}\left(S_{T} \times \mathbf{R}^{2 n}, \mathbf{R}\right)$ satisfying $\left\|H^{\prime \prime}\right\|_{C} \leq c$ for some $c>0$, the functional corresponding to the system

$$
\dot{x}=J H^{\prime}(t, x)
$$

is

$$
f(x)=\frac{1}{2} \int_{0}^{T} A x \cdot x d t-\int_{0}^{T} H(t, x) d t, \quad \forall x \in \operatorname{dom} A .
$$

Denote by $E_{\lambda}$ the spectral resolution of $A$ and write the orthogonal projection $P=\int_{-b}^{b} d E_{\lambda}$. Then $Z=P E$ is a subspace of $E$ with $\operatorname{dim} Z=2 d$ for some $d \in \mathbf{N}$. Via the saddle point reduction method of [AZ] (cf. also [Ch1, Ch2], as well as $\left[\right.$ Lo7]), if $b$ is chosen large enough, then there exist an injective map $u \in C^{1}(Z, E)$, 
$u(z)=z+v(z)$, where $P v(z)=0$, and a functional $a \in C^{2}(Z, \mathbf{R})$ defined by $a(z)=f(u(z))$, such that $z$ is a critical point of $a$ if and only if $u(z)$ is a critical point of $f$, i.e. a solution of (2.8).

Suppose that $z^{*}$ is a critical point of $a$. Let $x^{*}=u\left(z^{*}\right)$. We denote the Maslovtype index of $H^{\prime \prime}\left(t, x^{*}(t)\right)$ by $\left(i_{T}, \nu_{T}\right)=\left(i_{T}\left(x^{*}\right), \nu_{T}\left(x^{*}\right)\right)$ and call it the Maslovtype index of $x^{*}$. Denote the Morse indices of $a$ at $z^{*}$ by $m^{+}=m^{+}\left(z^{*}\right), m^{0}=$ $m^{0}\left(z^{*}\right)$, and $m^{-}=m^{-}\left(z^{*}\right)$, i.e. the multiplicities of the positive, zero, and negative eigenvalues of the matrix $a^{\prime \prime}\left(z^{*}\right)$. Let $2 d=\operatorname{dim} Z$.

Theorem 2.7 (Theorem 6 of [Lo1]). Under the above assumptions,

$$
m^{+}=d-i_{T}-\nu_{T}, \quad m^{0}=\nu_{T}, \quad m^{-}=d+i_{T} .
$$

Next we consider the homotopy invariance of the Maslov-type index theory.

Theorem 2.8. Given two paths $\gamma_{0}$ and $\gamma_{1}$ in $\mathcal{P}$, suppose $\gamma_{0} \sim \gamma_{1}$ in $\mathcal{P}$. Then

$$
\nu_{T}\left(\gamma_{0}\right)=\nu_{T}\left(\gamma_{1}\right) \quad \text { and } \quad i_{T}\left(\gamma_{0}\right)=i_{T}\left(\gamma_{1}\right) .
$$

Proof. Without loss of generality, we suppose $T=1$.

Because $\gamma_{0} \sim \gamma_{1}$ in $\mathcal{P}$, by Definition 2.2 there exists a map $\delta \in C([0,1], \mathcal{P})$ such that $\delta_{0}(\cdot)=\gamma_{0}(\cdot), \delta_{1}(\cdot)=\gamma_{1}(\cdot), \delta_{s}(0)=I$, and $\nu_{1}\left(\delta_{s}\right)$ is constant for $0 \leq s \leq 1$. If $\nu_{1}\left(\delta_{s}\right)=\nu_{1}\left(\gamma_{0}\right)=0$, then (2.10) was proved by Conley and Zehnder for the case $n \geq 2$ in [CZ] and by Long and Zehnder for the case $n=1$ in [LZ]. Therefore here we only need to prove $(2.10)$ when

$$
\nu_{1}\left(\delta_{s}\right)=\nu_{1}\left(\gamma_{0}\right)>0, \quad \forall 0 \leq s \leq 1 .
$$

Define

$$
B_{s}(t)=-J \dot{\delta}_{s}(t) \delta_{s}^{-1}(t), \quad \forall(s, t) \in[0,1]^{2} .
$$

Then $B_{s} \in C\left(S_{1}, \mathcal{L}_{s}\left(\mathbf{R}^{2 n}\right)\right)$ and $\delta_{s}:[0,1] \rightarrow \mathrm{Sp}(2 n)$ is the fundamental solution of the linear Hamiltonian system

$$
\dot{y}=J B_{s}(t) y .
$$

Define $\left\langle B_{s} x, x\right\rangle_{L^{2}}=\int_{0}^{1} B_{s}(t) x \cdot x d t$ for $x \in L^{2}\left(S_{1}, \mathbf{R}^{2 n}\right)$. By the saddle point reduction method of [AZ] and the compactness of the interval $[0,1]$, we obtain a finite-dimensional subspace $Z$ of $L^{2}\left(S_{1}, \mathbf{R}^{2 n}\right)$ with $\operatorname{dim} Z=2 d$ for some large integer $d>0$ and injective maps $u_{s}: Z \rightarrow \operatorname{dom}(A)$ such that the Morse indices $m_{s}^{+}, m_{s}^{0}$, and $m_{s}^{-}$of the functionals

$$
a_{s}(z)=\frac{1}{2}\left\langle\left(A-B_{s}\right) u_{s}(z), u_{s}(z)\right\rangle_{L^{2}}, \quad \forall z \in Z,
$$

at the origin satisfy the following equations:

$$
m_{s}^{+}=d-i_{1}\left(\delta_{s}\right)-\nu_{1}\left(\delta_{s}\right), \quad m_{s}^{0}=\nu_{1}\left(\delta_{s}\right), \quad m_{s}^{-}=d+i_{1}\left(\delta_{s}\right) .
$$

From (2.12) we obtain

$$
m_{s}^{0}=\nu_{1}\left(\delta_{s}\right)=\nu_{1}\left(\gamma_{0}\right), \quad \forall 0 \leq s \leq 1 .
$$

Claim. $m_{s}^{-}$and $m_{s}^{+}$are locally constant for $0 \leq s \leq 1$.

In fact, fix $r \in[0,1]$. When $s \in[0,1]$ is sufficiently close to $r$, by the perturbation theory on finite-dimensional spaces we have

$$
m_{r}^{-} \leq m_{s}^{-}, \quad m_{r}^{+} \leq m_{s}^{+} .
$$


Combining (2.17) with (2.16) yields

$$
2 d=m_{r}^{-}+m_{r}^{0}+m_{r}^{+} \leq m_{s}^{-}+m_{s}^{0}+m_{s}^{+}=2 d .
$$

Thus for $s$ sufficiently close to $r$ we must have

$$
m_{r}^{-}=m_{s}^{-}, \quad m_{r}^{+}=m_{s}^{+} .
$$

This proves the claim.

Thus, by $(2.15),(2.16)$ and the claim, we obtain that $i_{1}\left(\delta_{s}\right)$ is locally constant for $0 \leq s \leq 1$. This implies that $i_{1}\left(\delta_{s}\right)$ is globally constant for $0 \leq s \leq 1$, and completes the proof.

A direct consequence of Theorem 2.8 is that the Maslov-type index is invariant under conjugation in $\mathrm{Sp}(2 n)$.

Corollary 2.9. Given a path $\gamma$ in $\mathcal{P}$ and a matrix $M \in \operatorname{Sp}(2 n)$, define $\beta(t)=$ $M^{-1} \gamma(t) M$ for $0 \leq t \leq T$. Then

$$
\nu_{T}(\beta)=\nu_{T}(\gamma) \text { and } i_{T}(\beta)=i_{T}(\gamma) .
$$

Proof. Without loss of generality, we suppose $T=1$. Since $\operatorname{Sp}(2 n)$ is path connected, for $0 \leq s \leq 1$ let $A_{s}$ be a path in $\operatorname{Sp}(2 n)$ satisfying $A_{0}=I$ and $A_{1}=M$. For $(s, t) \in[0,1]^{2}$ define

$$
\delta_{s}(t)=A_{s}^{-1} \gamma(t) A_{s} \quad \text { and } \quad B_{s}(t)=-J \dot{\delta}_{s}(t) \delta_{s}^{-1}(t) .
$$

Then by the fact that $A_{s} \in \operatorname{Sp}(2 n)$ we obtain

$$
B_{s}(t)=-J A_{s}^{-1} \dot{\gamma}(t) \gamma^{-1}(t) A_{s}=A_{s}^{T}(-J) \dot{\gamma}(t) \gamma^{-1}(t) A_{s} .
$$

Thus $\delta_{s}$ is a path in $\mathcal{P}$ for $0 \leq s \leq 1$ satisfying $\delta_{0}=\gamma, \delta_{1}=\beta$, and

$$
\nu_{1}\left(\delta_{s}\right)=\operatorname{dim} \operatorname{ker}\left(A_{s}^{-1} \gamma(1) A_{s}-I\right)=\operatorname{dim} \operatorname{ker}(\gamma(1)-I) .
$$

Therefore $\delta_{s}$ for $0 \leq s \leq 1$ gives a homotopy of $\gamma$ and $\beta$ in $\mathcal{P}$, and (2.19) follows from Theorem 2.8 .

\section{Normal FORMS OF NONSINGUlar SYMPleCtiC MATRICES}

For $k \in \mathbf{N}$, we define the $k$ th order nonsingular matrix set of the symplectic group by

$$
\operatorname{Sp}(2 n)_{k}^{*}=\left\{M \in \operatorname{Sp}(2 n) \mid \operatorname{det}\left(M^{k}-I\right) \neq 0\right\} .
$$

Let $\operatorname{Sp}(2 n)_{k}^{0}=\operatorname{Sp}(2 n) \backslash \operatorname{Sp}(2 n)_{k}^{*}$. Note that $\operatorname{Sp}(2 n)_{k}^{*}$ is an open subset of $\operatorname{Sp}(2 n)$ in the topology induced from $\mathbf{R}^{2 n \times 2 n}$.

Definition 3.1. Two matrices $M$ and $M_{1}$ in $\operatorname{Sp}(2 n)$ are symplectically similar (and we write $\left.M \sim M_{1}\right)$ if there exists a matrix $A \in \mathrm{Sp}(2 n)$ such that $A^{-1} M A=M_{1}$.

Lemma 3.2. Suppose $M \in \operatorname{Sp}(2 n)_{k}^{*}$ for some $k \in \mathbf{N}, M_{1} \in \operatorname{Sp}(2 n)$ and $M \sim M_{1}$. Then both $M$ and $M_{1}$ belong to the same path-connected component of $\operatorname{Sp}(2 n)_{k}^{*}$.

Proof. Since $M \sim M_{1}$, by definition there exists a matrix $A \in \operatorname{Sp}(2 n)$ such that $A^{-1} M A=M_{1}$. By the fact that $\operatorname{Sp}(2 n)$ is path-connected, there exists a path $P:[0,1] \rightarrow \operatorname{Sp}(2 n)$ such that $P(0)=I$ and $P(1)=A$. From this we obtain that for every $s \in[0,1]$

$$
\operatorname{det}\left\{\left(P(s)^{-1} M P(s)\right)^{k}-I\right\}=\operatorname{det}\left(M^{k}-I\right) \neq 0 .
$$

Therefore $\gamma(s) \equiv P(s)^{-1} M P(s)$ is a path in $\operatorname{Sp}(2 n)_{k}^{*}$ connecting $M$ to $M_{1}$. 
Given any two symplectic matrices

$$
M_{1}=\left(\begin{array}{ll}
A_{1} & B_{1} \\
C_{1} & D_{1}
\end{array}\right)_{2 i \times 2 i}, \quad M_{2}=\left(\begin{array}{ll}
A_{2} & B_{2} \\
C_{2} & D_{2}
\end{array}\right)_{2 j \times 2 j},
$$

we define an operation $\diamond$-product of $M_{1}$ and $M_{2}$ to be the $2(i+j) \times 2(i+j)$ matrix $M_{1} \diamond M_{2}$ given by

$$
M_{1} \diamond M_{2}=\left(\begin{array}{cccc}
A_{1} & 0 & B_{1} & 0 \\
0 & A_{2} & 0 & B_{2} \\
C_{1} & 0 & D_{1} & 0 \\
0 & C_{2} & 0 & D_{2}
\end{array}\right) .
$$

Denote by $I_{i}$ the $i \times i$ identity matrix. When the dimension is clear, we will omit the subscript $i$. For $a \neq 0$ and $\theta \in \mathbf{R}$ define $2 \times 2$ matrices

$$
D(a)=\left(\begin{array}{cc}
a & 0 \\
0 & a^{-1}
\end{array}\right), \quad R(\theta)=\left(\begin{array}{cc}
\cos \theta & -\sin \theta \\
\sin \theta & \cos \theta
\end{array}\right) .
$$

Proposition 3.3. For $k \in 2 \mathrm{~N}-1$, each path-connected component of $\operatorname{Sp}(2 n)_{k}^{*}$ contains one of the following $2 n \times 2 n$ matrices:

$$
\left\{\begin{array}{l}
R\left(\theta_{1}\right) \diamond \cdots \diamond R\left(\theta_{\mu}\right) \\
R\left(\theta_{1}\right) \diamond \cdots \diamond R\left(\theta_{\mu}\right) \diamond D(2),
\end{array}\right.
$$

where $\theta_{i}=\left(2 t_{i}+\chi_{i}\right) \pi / k$, with integer $t_{i}$ satisfying $0 \leq t_{i} \leq k-1$, and $0<\chi_{i}<2$ for $1 \leq i \leq \mu$ and some integer $\mu$ satisfying $0 \leq \mu \leq n$.

For example, when $n=1$, there are $k+1$ such path-connected components in total.

Proof. We carry out the proof in two steps.

Step 1. Reduction to simple eigenvalues.

Let $M \in \operatorname{Sp}(2 n)_{k}^{*}$ and let $\lambda \in \sigma(M)$ be an eigenvalue with algebraic multiplicity $m>1$. Let $E_{\lambda}=\operatorname{ker}(M-\lambda I)^{m} \subset \mathbf{C}^{2 n}$ be the root vector space of $M$ belonging to $\lambda$. We choose a base $\left\{\xi_{1}, \ldots, \xi_{m}\right\}$ of $E_{\lambda}$ such that $(M-\lambda I) \xi_{j} \in \operatorname{span}\left\{\xi_{1}, \ldots, \xi_{j-1}\right\}$ for $2 \leq j \leq m$. Suppose $\lambda \notin \mathbf{R} \cup \mathbf{U}$. Choose a base $\left\{\eta_{1}, \ldots, \eta_{m}\right\}$ of $E_{\lambda^{-1}}$ such that $\left(\bar{J} \xi_{i}, \eta_{j}\right)_{\mathbf{C}^{2 n}}=\delta_{i, j}$. Here $(\cdot, \cdot)_{\mathbf{C}^{2 n}}$ denotes the inner product in $\mathbf{C}^{2 n}$. For $\varepsilon \geq 0$ define $B(\varepsilon) \in \operatorname{Sp}(2 n)$ such that $B(\varepsilon) \xi_{1}=(1+\varepsilon) \xi_{1}, B(\varepsilon) \bar{\xi}_{1}=(1+\varepsilon) \bar{\xi}_{1}, B(\varepsilon) \eta_{1}=$ $(1+\varepsilon)^{-1} \eta_{1}, B(\varepsilon) \bar{\eta}_{1}=(1+\varepsilon)^{-1} \bar{\eta}_{1}$, and $B(\varepsilon)=I$ on $E_{\mu} \oplus \operatorname{span}\left\{\xi_{i}, \eta_{i}, \bar{\xi}_{i}, \bar{\eta}_{i} \mid\right.$ $2 \leq i \leq m\}$ for $\mu \neq \lambda, \lambda^{-1}, \bar{\lambda}, \bar{\lambda}^{-1}$. Since $\operatorname{det}\left(M^{k}-I\right) \neq 0$ and $B(0)=I$, by continuity, there exists an $\varepsilon_{0}>0$ such that $\operatorname{det}\left((B(\varepsilon) M)^{k}-I\right) \neq 0$ for $|\varepsilon| \leq \varepsilon_{0}$, i.e. $B(\varepsilon) M \in \operatorname{Sp}(2 n)_{k}^{*}$. Similarly, when $\lambda \in \mathbf{R}$ or $\lambda \in \mathbf{U}$ is a multiple eigenvalue of $M$, we can also use small perturbations in $\operatorname{Sp}(2 n)_{k}^{*}$ to decrease the multiplicity of $\lambda$. Via such a method, we can continuously transform any matrix in $\operatorname{Sp}(2 n)_{k}^{*}$ to a matrix in $\operatorname{Sp}(2 n)_{k}^{*}$ with only simple eigenvalues. Therefore in the following, we only need to consider matrices in $\operatorname{Sp}(2 n)_{k}^{*}$ with only simple eigenvalues.

Step 2. Suppose $M \in \operatorname{Sp}(2 n)_{k}^{*}$ possessing only simple eigenvalues, and $\lambda \in \sigma(M)$.

Case 1. $\lambda \in \mathbf{C} \backslash(\mathbf{R} \cup \mathbf{U})$. Without loss of generality, we assume $|\lambda|<1$. Let $\omega(t)=[-t+\lambda(1-t)] / \lambda$. Then $\omega(0)=1, \omega(1)=-1 / \lambda$, and $B(\omega(t)-1) M$ changes the eigenvalue $\lambda$ to -1 when $t$ goes from 0 to 1 . We also notice that the eigenvalue $\lambda_{t}=-t+\lambda(1-t)$ of $B(\omega(t)-1) M$ satisfies $\lambda_{t}^{k} \neq 1$. This is because $\left|\lambda_{t}\right| \leq|t|+|\lambda|(1-t)<1$ when $0 \leq t<1$. 
Case 2. $\lambda<0$. Without loss of generality, we assume $\lambda<-1$. Then $B(\omega(t)-1) M$ changes the negative eigenvalue $\lambda$ to -1 in $\operatorname{Sp}(2 n)_{k}^{*}$ when $t$ goes from 0 to 1 .

Case 3. $M$ possesses two pairs of simple real positive eigenvalues, say $\lambda, \lambda^{-1}, \mu, \mu^{-1}$ with $\lambda>1$ and $\mu>1$. Define $\omega(t)=[t \lambda+(1-t) \mu] / \mu$. Then $B(\omega(t)-1) M$ changes $\mu$ and $\mu^{-1}$ to $\lambda$ and $\lambda^{-1}$ with $(\mu \omega(t))^{k} \neq 1$ when $t$ goes from 0 to 1 . Then this double eigenvalue pair $\lambda, \lambda^{-1}$ can be changed to $\lambda \pm i \varepsilon$ and $(\lambda \pm i \varepsilon)^{-1}$, and then can be changed to -1 continuously in $\operatorname{Sp}(2 n)_{k}^{*}$. Repeat this procedure for all positive eigenvalue pairs. If there still remains one more pair of positive eigenvalues, they can be changed to 2 and $1 / 2$ continuously in $\operatorname{Sp}(2 n)_{k}^{*}$.

Case 4. $\lambda \in \mathbf{U} \backslash \mathbf{R}$ and $\lambda^{k} \neq 1$. Let $e^{i \theta}=\lambda$ and $M \xi=e^{i \theta} \xi$. Then $M \bar{\xi}=e^{-i \theta} \bar{\xi}$. Now we adjust $\theta$ and $\xi$ so that $-(i J \xi, \xi)_{\mathbf{C}^{2 n}}=1 / 2$. This yields $M(\xi+\bar{\xi}, i(\xi-\bar{\xi}))=$ $(\xi+\bar{\xi}, i(\xi-\bar{\xi})) R(\theta)$. We can repeat this procedure for all such eigenvalues of $M$.

Through the above continuous transformations, we obtain that the given matrix $M$ is symplectically similar to some matrix of the form listed in (3.3). The proof is complete.

Proposition 3.4. For $k \in 2 \mathbf{N}$, each path-connected component of $\operatorname{Sp}(2 n)_{k}^{*}$ contains one of the following $2 n \times 2 n$ matrices:

$$
\left\{\begin{array}{l}
R\left(\theta_{1}\right) \diamond \cdots \diamond R\left(\theta_{\mu}\right) \diamond D(2) \diamond \cdots \diamond D(2), \\
R\left(\theta_{1}\right) \diamond \cdots \diamond R\left(\theta_{\mu}\right) \diamond D(2) \diamond \cdots \diamond D(2) \diamond D(-2),
\end{array}\right.
$$

where $\theta_{i}=\left(2 t_{i}+\chi_{i}\right) \pi / k \neq \pi$, with integer $t_{i}$ satisfying $0 \leq t_{i} \leq k-1$, and $0<\chi_{i}<2$ for $1 \leq i \leq \mu$ and some integer $\mu$ satisfying $0 \leq \mu \leq n$.

For example, when $n=1$, there are $k+2$ such path-connected components in total.

Proof. By Step 1 of the proof of Proposition 3.3, we may assume that $M \in \operatorname{Sp}(2 n)_{k}^{*}$ possesses only simple eigenvalues.

Case 1. Let $\lambda \in \sigma(M)$ and $\lambda \in \mathbf{C} \backslash(\mathbf{R} \cup \mathbf{U})$. Suppose $\lambda=|\lambda| e^{i \theta}$ with $|\lambda|>1$. Choose $\xi$ and $\eta$ such that $M \xi=\lambda \xi, M \eta=(1 / \lambda) \eta$, and $\xi^{\tau} J \eta=1$. Then

$$
M(\xi, \bar{\xi}, \eta, \bar{\eta})=(\xi, \bar{\xi}, \eta, \bar{\eta}) \operatorname{diag}\left(\lambda, \bar{\lambda}, \lambda^{-1}, \bar{\lambda}^{-1}\right) .
$$

Define $B(t)$ for $0 \leq t \leq 1$ by

$$
B(t)(\xi, \bar{\xi}, \eta, \bar{\eta})=(\xi, \bar{\xi}, \eta, \bar{\eta}) \operatorname{diag}\left(e^{i(-t \theta)}, e^{-i(-t \theta)}, e^{-i(-t \theta)}, e^{i(-t \theta)}\right),
$$

and set $B(t)=I$ on other root spaces of $M$. Then $B(t) M$ changes the eigenvalue group $\left\{\lambda, \bar{\lambda}, \lambda^{-1}, \bar{\lambda}^{-1}\right\}$ to $\left\{|\lambda|,|\lambda|,|\lambda|^{-1},|\lambda|^{-1}\right\}$ with $|\lambda|>1$ in $\operatorname{Sp}(2 n)_{k}^{*}$ continuously. As before, we then can change the eigenvalues to $\{2,2,1 / 2,1 / 2\}$.

Case 2. Suppose $\lambda \in \sigma(M)$. If $\lambda>1$, then $\lambda$ and $\lambda^{-1}$ can be changed to 2 and $1 / 2$. If $\lambda<-1$, then $\lambda$ and $\lambda^{-1}$ can be changed to -2 and $-1 / 2$. If there are two pairs of -2 and $-1 / 2$, then using the matrices $\operatorname{diag}\left(2 R((t-1) \pi), 2^{-1} R((t-1) \pi)\right)$ for $0 \leq t \leq 1$, they can be changed to $2,2,1 / 2,1 / 2$. Finally there remains at most one pair $-2,-1 / 2$.

Other cases can be treated as in the proof of Proposition 3.3, and this completes the proof. 
Definition 3.5. If matrices $M$ and $M_{1}$ belong to the same path-connected component of $\operatorname{Sp}(2 n)_{k}^{*}$ for some $k \in \mathbf{N}$, and $M_{1}$ is of the form (3.3) or (3.4), then $M_{1}$ is called a normal form of $M$. Denote by $\mu\left(M, M_{1}\right)$ the number $\mu$ of $M_{1}$ in (3.3) or (3.4). When there is no confusion, we simply write $\mu(M)$. Note that we do not need the uniqueness of the normal form of $M$ later.

\section{The iteration FORMUla FOR NONDEGEnERATE LINEAR HAMILTONIAN SYSTEMS}

In this section, we consider the linear Hamiltonian system

$$
\dot{y}=J B(t) y,
$$

where $B \in C\left(S_{T}, \mathcal{L}_{s}\left(\mathbf{R}^{2 n}\right)\right)$. Denote the fundamental solution of (4.1) by $\gamma:[0, T] \rightarrow$ $\operatorname{Sp}(2 n)$ with $\gamma(0)=I$. Then $\gamma \in \mathcal{P}$ as in $\S 2$. Denote the Maslov-type index of (4.1) by $\left(i_{T}, \nu_{T}\right)=\left(i_{T}(\gamma), \nu_{T}(\gamma)\right)$. For $k \in \mathbf{N}, B$ can also be viewed as defined on $S_{k T}$. Correspondingly we denote the Maslov-type index of (4.1) on $[0, k T]$ by $\left(i_{k T}, \nu_{k T}\right)$.

Define a path $\tilde{\gamma}:[0, k T] \rightarrow \operatorname{Sp}(2 n)$ by

$$
\tilde{\gamma}(t)=\gamma(t-j T) \gamma(T)^{j} \quad \text { for } j T \leq t \leq(j+1) T, 0 \leq j \leq k-1 .
$$

Then $\tilde{\gamma}$ is the fundamental solution of $(4.1)$ on $[0, k]$, and $\left(i_{k T}, \nu_{k T}\right)=\left(i_{k T}(\tilde{\gamma})\right.$, $\left.\nu_{k T}(\tilde{\gamma})\right)$.

The main result in this section is the following theorem:

Theorem 4.1. Suppose $\nu_{k T}=0$ for $k \in \mathbf{N}$. Then there exist an integer $\mu, 0 \leq$ $\mu \leq n$, and integers $t_{j}, 0 \leq t_{j} \leq k-1$ for $1 \leq j \leq \mu$, which are determined by a normal form of $\gamma(T)$ in (3.3) or (3.4), such that

$$
i_{k T}=k\left(i_{T}-\mu\right)+\sum_{j=1}^{\mu} 2 t_{j}+\mu .
$$

Moreover, if $\mu=n$, then the integers $\mu, i_{T}$, and $i_{k T}$ possess the same parity.

Remark 4.2. Note that there may be other normal forms in the same pathconnected component of $\operatorname{Sp}(2 n)_{k}^{*}$; thus the integers $\left\{\mu, t_{1}, \ldots, t_{k-1}\right\}$ need not be unique. What we are claiming is that for any choice of such integers determined by one of the normal forms of $\gamma(T)$, the iteration formula (4.3) always holds.

This section is devoted to the proof of Theorem 4.1. Without loss of generality, we assume $T=1$. We distinguish several cases according to the normal form of $\gamma(1)$.

Case 1. $\gamma(1)$ and $M$ belong to the same path-connected component of $\operatorname{Sp}(2 n)_{k}^{*}$, where we denote by $M$ the $2 n \times 2 n$ matrix

$$
M=R\left(\theta_{1}\right) \diamond \cdots \diamond R\left(\theta_{\mu}\right) \diamond D(2) \diamond \cdots \diamond D(2),
$$

where $\theta_{j}=\left(2 t_{j}+\chi_{j}\right) \pi / k, 0 \leq t_{j} \leq k-1,0<\chi_{i}<2$ for $1 \leq j \leq \mu, 0 \leq \mu \leq n$, and we suppose $n-\mu$ is even.

In this case, $M$ and $-I$, thus $\gamma(1)$ and $-I$, are in the same path-connected component of $\operatorname{Sp}(2 n)^{*}$. Therefore $i_{1}-n$ is even, and then $i_{1}-\mu$ is also even. Let

$$
2 h=i_{1}-\mu \text {. }
$$


Lemma 4.3. Under the above conditions there exists a homotopy $\gamma_{s}(t)$ for $0 \leq s \leq$ 1 and $0 \leq t \leq 1$, such that $\gamma_{0}=\gamma$,

$$
\gamma_{1}(t)=R\left(t\left(2 h \pi+\theta_{1}\right)\right) \diamond R\left(t \theta_{2}\right) \diamond \cdots \diamond R\left(t \theta_{\mu}\right) \diamond D\left(2^{t}\right) \diamond \cdots \diamond D\left(2^{t}\right),
$$

and

$$
\begin{aligned}
i_{1}\left(\gamma_{1}\right) & =i_{1}(\gamma), \\
i_{k}\left(\tilde{\gamma}_{1}\right) & =i_{k}(\tilde{\gamma}), \\
\nu_{k}\left(\tilde{\gamma}_{s}\right) & =0, \quad \forall 0 \leq s \leq 1 .
\end{aligned}
$$

Proof. Define the path $\gamma_{1}(t)$ for $0 \leq t \leq 1$ in $\operatorname{Sp}(2 n)_{k}^{*}$ by (4.6). Then $\gamma_{1}(1)=M$. Let $\alpha(t)$ for $0 \leq t \leq 1$ be a path in $\operatorname{Sp}(2 n)_{k}^{*}$ connecting $\gamma(1)$ to $M$. For $(s, t) \in[0,1]^{2}$ define

$$
\eta_{s}(t)= \begin{cases}\gamma(2 t /(2-s)), & s \leq 2-2 t \\ \alpha(s+2 t-2), & s \geq 2-2 t\end{cases}
$$

This gives a homotopy of $\gamma$ to $\alpha * \gamma$ in $\operatorname{Sp}(2 n)$ with end points in $\operatorname{Sp}(2 n)^{*}$.

Since $\tilde{\eta}_{s}(k)=\eta_{s}(1)^{k}=\alpha(s)^{k}$, we obtain

$$
\nu_{k}\left(\tilde{\eta}_{s}\right)=0, \quad \forall 0 \leq s \leq 1
$$

This implies (4.9).

By the definition of $h$, we have $i_{1}(\gamma)=2 h+\mu$. For the path $\gamma_{1}$, since $0<\theta_{j}<2 \pi$ for $1 \leq j \leq \mu$, the term $R\left(t\left(2 h \pi+\theta_{1}\right)\right)$ contributes $2 h+1$ to $i_{1}\left(\gamma_{1}\right)$, and the term $R\left(t \theta_{j}\right)$ contributes 1 to $i_{1}\left(\gamma_{1}\right)$ for $2 \leq j \leq \mu$. The term $D\left(2^{t}\right)$ contributes nothing to $i_{1}\left(\gamma_{1}\right)$. So we obtain $i_{1}\left(\gamma_{1}\right)=2 h+1+(\mu-1)=2 h+\mu$. Thus (4.7) holds.

To prove (4.8), for $a \in \mathbf{R}$ we define the $2 \times 2$ matrices

$$
D_{+}(a)=\left(\begin{array}{cc}
a & 0 \\
0 & a
\end{array}\right), \quad D_{-}(a)=\left(\begin{array}{cc}
0 & a \\
a & 0
\end{array}\right) .
$$

By direct computation we obtain

$$
\begin{aligned}
B_{1}(t) & =-J \dot{\gamma}_{1}(t) \gamma_{1}^{-1}(t) \\
& =D_{+}\left(2 h \pi+\theta_{1}\right) \diamond D_{+}\left(\theta_{2}\right) \diamond \cdots \diamond D_{+}\left(\theta_{\mu}\right) \diamond D_{-}(-\log 2) \diamond \cdots \diamond D_{-}(-\log 2) .
\end{aligned}
$$

Let

$$
\begin{aligned}
& \mathcal{P}_{k}=\left\{\beta \in C([0,1], \operatorname{Sp}(2 n)) \mid \beta(0)=I, \nu_{k}(\tilde{\beta})=0\right\}, \\
& \mathcal{P}_{k}^{\prime}=\left\{\beta \in C^{1}([0,1], \operatorname{Sp}(2 n)) \mid \beta(0)=I, \nu_{k}(\tilde{\beta})=0, \dot{\beta}(1)=\dot{\beta}(0) \beta(1)\right\} .
\end{aligned}
$$

As proved in [LZ] and [Lo7], since $\gamma, \gamma_{1} \in \mathcal{P}$, and $\gamma \sim \gamma_{1}$ in $\mathcal{P}_{k}$, then the homotopy can be chosen in $\mathcal{P}_{k}^{\prime}$. We denote this homotopy by $\gamma_{s}$.

We consider the linear system (4.1) in the space $L=L^{2}\left(S_{k}, \mathbf{R}^{2 n}\right)$. The path $\tilde{\gamma}:[0, k] \rightarrow \operatorname{Sp}(2 n)$ gives the fundamental solution of $(4.1)$ on $[0, k]$. The homotopy $\gamma_{s}$ connects $\gamma$ to $\gamma_{1}$. Define

$$
B_{s}(t)=-J \dot{\gamma}_{s}(t) \gamma_{s}^{-1}(t) \quad \text { for }(s, t) \in[0,1]^{2} .
$$

Note that $B_{s}(t)$ must be symmetric for $0 \leq s \leq 1$. Then $\tilde{\gamma}_{s}(t)$ for $0 \leq t \leq k$ is the fundamental solution of the linear system

$$
\dot{y}=J B_{s}(t) y
$$

and $\nu_{k}\left(\tilde{\gamma}_{s}\right)=0$. 
By the saddle point reduction method of $[\mathrm{AZ}]$ and the compactness of the unit interval, we obtain the same finite-dimensional space $Z$ for all $s \in[0,1]$ with sufficiently large $m_{0} \in \mathbf{N}$ :

$$
\begin{aligned}
Z & =\mathbf{R}^{2 n} \oplus\left(\bigoplus_{m=1}^{m_{0}} E_{m}\right), \\
E_{m} & =\left\{\cos \left(\frac{2 m \pi t}{k}\right) \xi+\sin \left(\frac{2 m \pi t}{k}\right) \eta \mid \xi, \eta \in \mathbf{R}^{2 n}\right\},
\end{aligned}
$$

and we also obtain functions $a_{s}: Z \rightarrow \mathbf{R}$, and maps $u_{s}: Z \rightarrow L$ such that

$$
a_{s}(z)=\left\langle\left(A-B_{s}\right) u_{s}(z), u_{s}(z)\right\rangle \quad \forall z \in Z .
$$

Here $B_{s}$ is the operator on $L$ defined by

$$
\left\langle B_{s} x, x\right\rangle=\int_{0}^{k} B_{s}(t) x(t) \cdot x(t) d t \quad \forall x \in L
$$

and $B_{0}=B$. Since $\nu_{k}\left(\tilde{\gamma}_{s}\right)=0$, the inverse operator $\left(A-B_{s}\right)^{-1}$ exists on the space $Z$, and $z=0$ is an isolated critical point of all $a_{s}$. We denote the Morse indices of $a_{s}$ at $z=0$ by $m_{s}^{-}, m_{s}^{0}=0$, and $m_{s}^{+}$. Denote $2 d=\operatorname{dim} Z$. Using Conley index theory, in [LZ] and [Lo7] it is proved that

$$
m_{s}^{-}=m_{0}^{-}, \quad m_{s}^{0}=0, \quad m_{s}^{+}=m_{0}^{+}, \quad \forall s \in[0,1] .
$$

By Theorem 2.7,

$$
m_{s}^{-}=d+i_{k}\left(\tilde{\gamma}_{s}\right), \quad m_{s}^{0}=0, \quad m_{s}^{+}=d-i_{k}\left(\tilde{\gamma}_{s}\right), \quad \forall s \in[0,1] .
$$

This implies (4.8).

The proof is complete.

Proof of the iteration formula (4.2) in Case 1.

By Lemma 4.3, it suffices to compute $i_{k}\left(\tilde{\gamma}_{1}\right)$. Note that $\tilde{\gamma}_{1}(t)=\gamma_{1}(t)$ for $0 \leq t \leq$ $k$. From the definition (4.6) of $\gamma_{1}$ and $\theta_{j}=\left(2 t_{j}+\chi_{j}\right) \pi / k$ for $1 \leq j \leq \mu$, we obtain

$$
\gamma_{1}(k)=R\left(k\left(2 h \pi+\theta_{1}\right)\right) \diamond R\left(k \theta_{2}\right) \diamond \cdots \diamond R\left(k \theta_{\mu}\right) \diamond D\left(2^{k}\right) \diamond \cdots \diamond D\left(2^{k}\right),
$$

where $h$ is defined by (4.5). Thus the rotation number of $\gamma_{1}$ on $[0, k]$ is

$$
\begin{aligned}
\Delta_{k}\left(\gamma_{1}\right) & =k\left(2 h \pi+\theta_{1}\right)+k\left(\theta_{2}+\cdots+\theta_{\mu}\right) \\
& =\left(2 h k+\sum_{j=1}^{\mu}\left(2 t_{j}+\chi_{j}\right)\right) \pi \\
& =\left(2 h k+\sum_{j=1}^{\mu} 2 t_{j}+\sum_{j=1}^{\mu} \chi_{j}\right) \pi .
\end{aligned}
$$

At this stage, since $0<\chi_{j}<2$, in the computation of indices, without loss of generality, we may choose $\chi_{j}=1$. Thus we obtain

$$
\begin{aligned}
i_{k}\left(\gamma_{1}\right) & =2 h k+\sum_{j=1}^{\mu} 2 t_{j}+\sum_{j=1}^{\mu} 1 \\
& =k\left(i_{1}-\mu\right)+\sum_{j=1}^{\mu} 2 t_{j}+\mu .
\end{aligned}
$$


Here we have used (4.5). Combining this equality with Lemma 4.3 yields (4.3).

Here we especially notice that in this case the integers $\mu, i_{1}$, and $i_{k}$ have the same parity.

Case 2. Suppose all the conditions in Case 1 hold except that $n-\mu$ is odd. Then $\mu<n$.

Since $n-\mu$ is odd, the matrix $M$ defined in (4.4) possesses an eigenvalue pair $\{2,1 / 2\}$ of odd multiplies. Thus in $\operatorname{Sp}(2 n)^{*}$ one can connect $M$ to $(-I) \diamond D(2)$. This implies $i_{1}-(n-1)$ is even. Therefore $\mu$ and $i_{1}$ have the same parity. By the proof of Case 1, we obtain the required formula (4.3):

$$
i_{k}=i_{k}\left(\gamma_{1}\right)=k\left(i_{1}-\mu\right)+\sum_{j=1}^{\mu} 2 t_{j}+\mu .
$$

This formula shows that $i_{k}$ and $\mu$ also have the same parity.

Case 3. $\gamma(1)$ and $M$ belong to the same connected component of $\operatorname{Sp}(2 n)_{k}^{*}$, where we denote by $M$ the $2 n \times 2 n$ matrix

$$
M=R\left(\theta_{1}\right) \diamond \cdots \diamond R\left(\theta_{\mu}\right) \diamond D(2) \diamond \cdots \diamond D(2) \diamond D(-2),
$$

where $\theta_{j}=\left(2 t_{j}+\chi_{j}\right) \pi / k, 0 \leq t_{j} \leq k-1,0<\chi_{j}<2$ for $1 \leq j \leq \mu, 0 \leq \mu \leq n$.

Note that in this case we must have $\mu<n$. Let $h=i_{1}-\mu-1$.

When $k$ is odd, -1 is not a root of unity. So $D(-2)$ can be transformed through $D(-1)$ to $R(\theta)$ for some $\theta$ near $\pi$. Thus this case can be reduced to the above two cases. Therefore we only consider the case when $k$ is even. Similarly to Lemma 4.3, we obtain

Lemma 4.4. Suppose $k$ is even, and $\theta_{j} \neq \pi$ in (4.25). Under the above conditions there exists a homotopy $\gamma_{s}(t)$ for $0 \leq s \leq 1$ and $0 \leq t \leq 1$, such that $\gamma_{0}=\gamma$,

$$
\begin{aligned}
\gamma_{1}(t)=R\left(t\left(h \pi+\theta_{1}\right)\right) & \diamond R\left(t \theta_{2}\right) \diamond \cdots \diamond R\left(t \theta_{\mu}\right) \\
& \diamond D\left(2^{t}\right) \diamond \cdots \diamond D\left(2^{t}\right) \diamond D\left(2^{t}\right) R(t \pi),
\end{aligned}
$$

and

$$
\begin{aligned}
i_{1}\left(\gamma_{1}\right) & =i_{1}(\gamma), \\
i_{k}\left(\tilde{\gamma}_{1}\right) & =i_{k}(\tilde{\gamma}), \\
\nu_{k}\left(\tilde{\gamma}_{s}\right) & =0 \quad \forall 0 \leq s \leq 1 .
\end{aligned}
$$

Since $D(2) R(\pi)=R(\pi) D(2)$, viewing $\gamma_{1}$ as a path defined on $[0, k]$, we have $\tilde{\gamma}_{1}(j)=\gamma_{1}(1)^{j}=\gamma_{1}(j)$ for $1 \leq j \leq k$. Thus $i_{k}\left(\tilde{\gamma}_{1}\right)=i_{k}\left(\gamma_{1}\right)$.

From (4.26) we see that the rotation number of $\gamma_{1}$ on $[0,1]$ is

$$
\Delta_{1}\left(\gamma_{1}\right)=\left(h \pi+\theta_{1}\right)+\left(\theta_{2}+\cdots+\theta_{\mu}\right)+\pi .
$$

At this stage, since $0<\theta_{j}<2 \pi$, in the computation of indices, without loss of generality, we may choose $\theta_{j}=\pi$. Thus we obtain

$$
i_{1}\left(\gamma_{1}\right)=h+\mu+1
$$

Similarly, by (4.26) the rotation number of $\gamma_{1}$ on $[0, k]$ is

$$
\begin{aligned}
\Delta_{k}\left(\gamma_{1}\right) & =k\left(h \pi+\theta_{1}\right)+k\left(\theta_{2}+\cdots+\theta_{\mu}\right)+k \pi \\
& =\left((h+1) k+\sum_{j=1}^{\mu} 2 t_{j}+\sum_{j=1}^{\mu} \chi_{j}\right) \pi .
\end{aligned}
$$


At this stage, since $0<\chi_{j}<2$, in the computation of indices, without loss of generality, we may choose $\chi_{j}=1$. Thus we obtain

$$
\begin{aligned}
i_{k}\left(\gamma_{1}\right) & =(h+1) k+\sum_{j=1}^{\mu} 2 t_{j}+\sum_{j=1}^{\mu} 1 \\
& =k\left(i_{1}-\mu\right)+\sum_{j=1}^{\mu} 2 t_{j}+\mu .
\end{aligned}
$$

Here we have used $h=i_{1}-\mu-1$. Combining this equality with Lemma 4.4 yields (4.3) in Case 3.

We have studied all the cases in (3.3) and (3.4) and thus completed the proof of Theorem 4.1.

\section{Perturbations of eigenvalues AWAy From 1}

For $k \in \mathbf{N}$, in order to establish the $k$ th iteration formula for degenerate linear Hamiltonian systems, we need to perturb the end point of the path $\gamma$ in $\operatorname{Sp}(2 n)$ corresponding to the fundamental solution of this system defined on the time interval $[0, T]$. Therefore we need to study perturbations of a singular symplectic matrix on its root spaces belonging to eigenvalues which are $k$ th roots of unity.

For $k \in \mathbf{N}$, suppose $M \in \operatorname{Sp}(2 n)$ satisfies $\operatorname{det}\left(M^{k}-I\right)=0$. Then $M$ possesses eigenvalues $1,-1$, or $e^{ \pm \sqrt{-1}(j \pi / k)}$ with $1 \leq j \leq k-1$. Since the root vector spaces of these different eigenvalues are symplectically orthogonal to each other, it suffices to study perturbations of $M$ on each root vector space separately. In this section we study the case of eigenvalue 1, and the other cases will be studied in the next two sections respectively.

Definition 5.1. $E, F \subset \mathbf{R}^{2 n}$ are symplectically orthogonal to each other (denoted by $E \perp F)$ if $\xi^{T} J \eta=0$ for all $\xi \in E$ and $\eta \in F$. Two vectors $\xi$ and $\eta$ form a normal pair if $\xi^{T} J \eta=1$, and each called the partner of the other.

Suppose $M \in \operatorname{Sp}(2 n)$ possesses the eigenvalue 1. Since perturbations of eigenvalues away from 1 have been carefully studied in [Lo1, Lo2] and [Lo9], we are very sketchy in this section.

Denote by $E_{1}$ the root vector space belonging to the eigenvalue 1 of $M$. Then

$$
\mathbf{R}^{2 n}=E_{1} \oplus E_{1}^{\perp} .
$$

Choose a symplectic base $\Delta_{1}=\left\{\xi_{i} \mid 1 \leq i \leq 2 m\right\}$ of $E_{1}$ with $2 m=\operatorname{dim} E_{1}$, then extend it to a symplectic base $\Delta=\left\{\xi_{i} \mid 1 \leq i \leq 2 n\right\}$ of $\mathbf{R}^{2 n}$. Then there exists a matrix $M_{1} \in \operatorname{Sp}(2 m)$ such that

$$
M\left(\xi_{1}, \ldots, \xi_{2 m}\right)=\left(\xi_{1}, \ldots, \xi_{2 m}\right) M_{1} .
$$

Thus there exists a matrix $P \in \operatorname{Sp}(2 n)$ such that $P^{-1} M P=M_{1}$ on $P^{-1} E_{1}$. Note that $\sigma\left(\left.P^{-1} M P\right|_{P^{-1} E_{1}^{\perp}}\right)=\sigma\left(\left.M\right|_{E_{1}^{\perp}}\right)$.

In [Lo1, Lo2] and [Lo9], using the rotation matrices defined in $\S 2$, it is proved that there exist an integer $q$ with $1 \leq q \leq m$, a strictly increasing subsequence $\left\{m_{1}, \ldots, m_{q}\right\}$ of $\{1, \ldots, m\}$, and $\theta_{0}>0$ sufficiently small such that for any $\tau \in$ $[-1,1] \backslash\{0\}$, if we define $2 m \times 2 m$ matrices

$$
G(\tau) \equiv R_{m_{1}}\left(\tau \theta_{0}\right) \cdots R_{m_{q}}\left(\tau \theta_{0}\right),
$$


then the following relations hold:

$$
\begin{gathered}
P^{-1} M P(G(\tau) \diamond I) \in \operatorname{Sp}(2 n)^{*}, \\
P^{-1} M P\left(G(\tau) R_{m_{j}}\left(-\tau \theta_{0}\right) \diamond I\right) \in \operatorname{Sp}(2 n)^{0} \quad \text { for } 1 \leq j \leq q .
\end{gathered}
$$

Thus when $|\tau|>0$ is small, there exists a matrix $P \in \operatorname{Sp}(2 n)$ such that

$$
P^{-1} M P(G(\tau) \diamond I)=P^{-1} M P
$$

on $P^{-1} E_{1}^{\perp}$, and $P^{-1} M P(G(\tau) \diamond I)$ perturbs the eigenvalue 1 of $M$ to eigenvalues of $P^{-1} M P(G(\tau) \diamond I)$ away from 1 .

As we mentioned in $\S 2$, such perturbations are used in [Lo1] and [Lo9] to define the Maslov-type index for paths in $\mathcal{P}$ with end point $P^{-1} M P$.

\section{Perturbations of eigenvalues away from -1}

Suppose $M \in \operatorname{Sp}(2 n)$ possesses the eigenvalue -1 . In this section we study perturbations on $M$ to change -1 to nearby eigenvalues on $\mathbf{U} \backslash \mathbf{R}$ or $\mathbf{R} \backslash\{-1\}$.

In [LD] the following normal forms $M_{1} \in \mathrm{Sp}(2 k)$ for symplectic matrices possessing the eigenvalue -1 were introduced:

Normal form $1 . M_{1} \in \mathrm{Sp}(2)$ defined by

$$
M_{1}=\left(\begin{array}{cc}
-1 & 1 \\
0 & -1
\end{array}\right),\left(\begin{array}{cc}
-1 & -1 \\
0 & -1
\end{array}\right) \text {, or }\left(\begin{array}{cc}
-1 & 0 \\
0 & -1
\end{array}\right) \text {. }
$$

Normal form 2. $M_{1} \in \operatorname{Sp}(2 k)$ with $k \geq 2$ defined by

$$
M_{1}=\left(\begin{array}{cc}
A & B \\
0 & C
\end{array}\right)
$$

where $A, B$, and $C$ are $k \times k$ matrices, $A$ is a $k \times k$ Jordan block form matrix of eigenvalue -1 :

$$
A=\left(\begin{array}{cccccc}
-1 & 1 & 0 & \ldots & 0 & 0 \\
0 & -1 & 1 & \ldots & 0 & 0 \\
0 & 0 & -1 & \ldots & 0 & 0 \\
. & . & . & \ldots & . & . \\
. & . & . & \ldots & . & . \\
0 & 0 & 0 & \ldots & -1 & 1 \\
0 & 0 & 0 & \ldots & 0 & -1
\end{array}\right)
$$

$B$ and $C$ are lower triangular matrices of the following forms with $b_{i} \in \mathbf{R}, 1 \leq i \leq k$ :

$$
B=\left(\begin{array}{cccccc}
b_{1} & 0 & 0 & \ldots & 0 & 0 \\
b_{2} & b_{2} & 0 & \ldots & 0 & 0 \\
b_{3} & b_{3} & b_{3} & \ldots & 0 & 0 \\
\cdot & \cdot & \cdot & \ldots & \cdot & \cdot \\
\cdot & \cdot & \cdot & \ldots & \cdot & \cdot \\
b_{k-1} & b_{k-1} & b_{k-1} & \ldots & b_{k-1} & 0 \\
b_{k} & b_{k} & b_{k} & \ldots & b_{k} & b_{k}
\end{array}\right),
$$




$$
C=\left(\begin{array}{cccccc}
-1 & 0 & 0 & \ldots & 0 & 0 \\
-1 & -1 & 0 & \ldots & 0 & 0 \\
-1 & -1 & -1 & \ldots & 0 & 0 \\
\cdot & \cdot & \cdot & \ldots & . & \cdot \\
\cdot & \cdot & \cdot & \ldots & \cdot & \cdot \\
-1 & -1 & -1 & \ldots & -1 & 0 \\
-1 & -1 & -1 & \ldots & -1 & -1
\end{array}\right)
$$

In $[\mathrm{LD}]$ the following result on the normal forms of symplectic matrices with eigenvalue -1 is proved.

Theorem 6.1. Suppose $M \in \mathrm{Sp}(2 n)$ possesses the eigenvalue -1 . Denote by $E_{-1}$ the invariant root vector space of $M$ belonging to the eigenvalue -1 . Then there exist $P \in \operatorname{Sp}(2 n)$ and $p \in \mathbf{N}$ such that

$$
P^{-1} M P=M_{1} \diamond \cdots \diamond M_{p} \diamond M_{0} .
$$

Here $M_{i} \in \operatorname{Sp}\left(2 k_{i}\right)$ for $0 \leq i \leq p$. Moreover, $2 \sum_{i=0}^{p} k_{i}=2 k_{0}+\operatorname{dim} E_{-1}=2 n$, $k_{0} \geq 0$, and $-1 \notin \sigma\left(M_{0}\right)$. For $1 \leq i \leq p, k_{i} \geq 1$, each $M_{i}$ is a normal form matrix of eigenvalue -1 defined by (6.1)-(6.5).

Based upon this theorem, we study first the perturbations of the normal form matrix $M_{1} \in \mathrm{Sp}(2 k)$ of eigenvalue -1 defined by (6.1)-(6.5). We distinguish three cases.

Case 1. $k \in \mathbf{N}$ is even.

$1^{\circ}$. We multiply $M_{1}$ by the $2 k \times 2 k$ matrix $G(\tau)=\operatorname{diag}(R(\tau), \ldots, R(\tau))$ with $\tau \neq 0$, and obtain

$$
\operatorname{det}\left(M_{1} G(\tau)-\lambda I\right)=\left(\lambda^{2}+(2 \cos \tau-\sin \tau) \lambda+1\right)^{k} .
$$

Thus we obtain the complex conjugate eigenvalues

$$
\lambda^{ \pm 1}=\frac{1}{2}\left\{(\sin \tau-2 \cos \tau) \pm \sqrt{-2 \sin (2 \tau)-3 \sin ^{2} \tau}\right\} .
$$

When $0<\tau<\pi / 2$, they satisfy $\lambda^{ \pm 1} \in \mathbf{U} \backslash \mathbf{R}$, and are $k$-multiple eigenvalues of the $2 k \times 2 k$ matrix $M_{1} G(\tau)$.

$2^{\circ}$. From the formula (6.7), if we choose $-\tau \in(0, \pi / 2)$ sufficiently small, the eigenvalue -1 is perturbed to $k$-multiple negative eigenvalues $\lambda^{ \pm 1}$ given by (6.8).

Therefore we have proved that $M_{1} G(\tau)$ perturbs the eigenvalue -1 of $M_{1}$ to the nearby eigenvalue $\lambda^{ \pm 1}$ on the unit circle for small $\tau>0$, or to the nearby eigenvalues $\lambda^{ \pm 1}$ on $\mathbf{R} \backslash\{-1\}$ for small $-\tau>0$. Note that in this subcase, the perturbation has nothing to do with $b_{k}$ in (6.4)

Case 2. $k \geq 3$ is odd and $b_{k} \neq 0$.

$1^{\circ}$. We multiply $M_{1}$ by the $2 k \times 2 k$ matrix $G(\tau, \theta)=\operatorname{diag}(R(\tau), \ldots, R(\tau)) \diamond R(\theta)$ with $\tau$ and $\theta \neq 0$. Then we obtain

$$
\begin{aligned}
& \operatorname{det}\left(M_{1} G(\tau, \theta)-\lambda I\right) \\
& \quad=\left(\lambda^{2}+(2 \cos \tau-\sin \tau) \lambda+1\right)^{k}\left(\lambda^{2}+\left(2 \cos \theta-b_{k} \sin \theta\right) \lambda+1\right),
\end{aligned}
$$

where $b_{k}$ is defined in (6.4) for $B$. Thus for $0<\tau<\pi / 2$ and small $\theta \neq 0$ such that

$$
Q\left(b_{k}, \theta\right) \equiv 2 b_{k} \sin (2 \theta)+\left(4-b_{k}^{2}\right) \sin ^{2} \theta>0,
$$


we obtain the complex conjugate eigenvalues $\lambda^{ \pm 1}$ given by (6.8) and

$$
\rho^{ \pm 1}=\frac{1}{2}\left\{\left(b_{k} \sin \theta-2 \cos \theta\right) \pm \sqrt{-Q\left(b_{k}, \theta\right)}\right\} .
$$

They satisfy $\lambda^{ \pm 1}$ and $\rho^{ \pm 1} \in \mathbf{U} \backslash \mathbf{R}$. Note that $\lambda^{ \pm 1}$ are $k$-multiple eigenvalues and $\rho^{ \pm 1}$ are simple eigenvalues of the $2 k \times 2 k$ matrix $M_{1} G(\tau, \theta)$.

$2^{\circ}$. By (6.8) and (6.10), if we choose $-\tau>0$ sufficiently small and $|\theta|>0$ small such that $Q\left(b_{k}, \theta\right)<0$, the eigenvalue -1 is perturbed to nearby negative eigenvalues $\lambda^{ \pm 1}$ and $\rho^{ \pm 1}$ on $\mathbf{R} \backslash\{-1\}$.

Therefore we have proved that $M_{1} G(\tau, \theta)$ perturbs the eigenvalue -1 of $M_{1}$ to nearby eigenvalues on the unit circle for small $\tau>0$ and small $\theta \neq 0$ satisfying $Q\left(b_{k}, \theta\right)>0$, or to nearby eigenvalues on $\mathbf{R} \backslash\{-1\}$ for small $-\tau>0$ and small $\theta \neq 0$ satisfying $Q\left(b_{k}, \theta\right)<0$. Note that in this subcase, the perturbation needs to be chosen carefully according to whether $b_{k}>0$ or $b_{k}<0$.

Case 3. $k \geq 3$ is odd and $b_{k}=0$.

$1^{\circ}$. Note that when $b_{k}=0$ the perturbation in $1^{\circ}$ of Case 2 always satisfies $Q\left(b_{k}, \theta\right)>0$ and also perturbs the eigenvalue -1 of $M_{1}$ to nearby eigenvalues $\lambda^{ \pm 1}$ and $\rho^{ \pm 1}$ on $\mathbf{U} \backslash \mathbf{R}$ for small $\tau>0$ and small $\theta \neq 0$.

$2^{\circ}$. In order to perturb the eigenvalue -1 of $M_{1}$ to nearby eigenvalues on $\mathbf{R} \backslash\{-1\}$, we multiply $M_{1}$ by the $2 k \times 2 k$ matrix $G(\tau, \theta)=\operatorname{diag}(R(\tau), \ldots, R(\tau)) \diamond$ $D\left(2^{\theta}\right)$ with $\tau$ and $\theta \neq 0$. Then we obtain

$$
\begin{aligned}
\operatorname{det}( & \left.M_{1} G(\tau, \theta)-\lambda I\right) \\
\quad & =\left(\lambda^{2}+(2 \cos \tau-\sin \tau) \lambda+1\right)^{k}\left(2^{\theta}+\lambda\right)\left(2^{-\theta}+\lambda\right) .
\end{aligned}
$$

Thus for small $-\tau \in(0, \pi / 2)$ and small $\theta \neq 0$, we obtain the negative eigenvalues $\lambda^{ \pm 1}$ given by $(6.8)$ and

$$
\rho^{ \pm 1}=-2^{ \pm \theta}
$$

They satisfy $\lambda^{ \pm 1}$ and $\rho^{ \pm 1} \in \mathbf{R} \backslash\{-1\}$ near -1 . Note that $\lambda^{ \pm 1}$ are $k$-multiple eigenvalues and $\rho^{ \pm 1}$ are simple eigenvalues of the $2 k \times 2 k$ matrix $M_{1} G(\tau, \theta)$.

Therefore we have proved that $M_{1} G(\tau, \theta)$ perturbs the eigenvalue -1 of $M_{1}$ to nearby eigenvalues on the unit circle for small $\tau>0$ and small $\theta \neq 0$, or to nearby eigenvalues on $\mathbf{R} \backslash\{-1\}$ for small $-\tau>0$ and small $\theta \neq 0$.

Case 4. $k=1$.

In this case, $M_{1}$ is given by (6.1). Write

$$
M_{1}=\left(\begin{array}{cc}
-1 & b \\
0 & -1
\end{array}\right) \text {. }
$$

Subcase $1 . b=0$. We have $M_{1}=-I$. Then the geometric multiplicity of the eigenvalue $-1 \in \sigma\left(M_{1}\right)$ is 2 . When $\tau$ perturbs away from $\tau=0$, the corresponding eigenvalues of $M_{1} R(\tau)=R(\pi+\tau)$ are changed to $e^{ \pm \sqrt{-1}(\pi+\tau)} \in \mathbf{U} \backslash \mathbf{R}$ near -1 . For any $m \in \mathbf{N}$, when $0<\tau<\pi / m$, we obtain

$$
\pi+\tau=\frac{2 t+\chi}{2 m} \pi, \quad \text { with } t=m \geq 1 \text { and } 0<\chi=\frac{2 \tau}{\pi}<2 .
$$

On the other hand, when $\tau$ perturbs away from $\tau=0$, the corresponding eigenvalues of $M_{1} D\left(2^{\tau}\right)=D\left(-2^{\tau}\right)$ are changed to $-2^{ \pm \tau} \in \mathbf{R} \backslash\{-1\}$ near -1 . 
Subcase 2. $b=1$. Then the geometric multiplicity of the eigenvalue $-1 \in \sigma\left(M_{1}\right)$ is 1. We write the $2 \times 2$ matrix $M_{1}$ in polar form with $\pi / 2<\alpha<3 \pi / 2$ :

$$
M_{1}=\left(\begin{array}{cc}
-1 & b \\
0 & -1
\end{array}\right)=\left(\begin{array}{cc}
r & z \\
z & \frac{1+z^{2}}{r}
\end{array}\right)\left(\begin{array}{cc}
\cos \alpha & -\sin \alpha \\
\sin \alpha & \cos \alpha
\end{array}\right) .
$$

Then this yields $2 \tan \alpha=b>0$. Thus $\pi<\alpha<3 \pi / 2$. On the other hand, when $\tau>0$ is sufficiently small that $\pi<\alpha+\tau<3 \pi / 2$, the corresponding eigenvalues of

$$
M_{1} R(\tau)=\left(\begin{array}{cc}
r & z \\
z & \frac{1+z^{2}}{r}
\end{array}\right)\left(\begin{array}{cc}
\cos (\alpha+\tau) & -\sin (\alpha+\tau) \\
\sin (\alpha+\tau) & \cos (\alpha+\tau)
\end{array}\right)
$$

are

$$
\lambda^{ \pm 1}=\left(\frac{1}{2} b \sin \tau-\cos \tau\right) \pm \sqrt{-\frac{b}{2} \sin (2 \tau)-\left(1-b^{2} / 2\right) \sin ^{2} \tau}
$$

satisfying $\lambda^{ \pm 1} \in \mathbf{U} \backslash \mathbf{R}$. Thus for any $m \in \mathbf{N}$, if $\tau \in(0, \pi / m)$ is so small that $\pi<\alpha+\tau<3 \pi / 2$, then we obtain

$$
\alpha+\tau=\frac{2 t+\chi}{2 m} \pi, \quad \text { with } t=m \geq 1 \text { and } 0<\chi=\frac{2 m \tau}{\pi}<2 .
$$

Note also that when $\tau<0$ is small, the eigenvalue -1 of $M_{1}$ are perturbed to eigenvalues $\lambda^{ \pm 1} \in \mathbf{R} \backslash\{-1\}$ near -1 .

Subcase 3. $b=-1$. Then the geometric multiplicity of the eigenvalue $-1 \in \sigma\left(M_{1}\right)$ is 1 .

$1^{\circ}$. We write the $2 \times 2$ matrix $M_{1}$ in its polar form (6.14). This yields $\sin ^{2} \alpha+$ $\tan \alpha\left(1+\sin ^{2} \alpha\right)=b<0$. Thus $\pi / 2<\alpha<\pi$. On the other hand, when $\tau<0$ is sufficiently small that $\pi / 2<\alpha+\tau<\pi$, the corresponding eigenvalues $\lambda^{ \pm 1}$ of (6.15) are still given by (6.16), and $\lambda^{ \pm 1} \in \mathbf{U} \backslash \mathbf{R}$. Fix $m \in \mathbf{N}$. If $[m \alpha / \pi]=m \alpha / \pi$, we must have $m \geq 2$. Thus, whenever $-\tau>0$ is small enough,

$$
\alpha+\tau=\frac{2 t+\chi}{2 m} \pi, \quad \text { with } t=\left[\frac{m \alpha}{\pi}\right]-1 \geq 1,0<\chi=2+\frac{2 m \tau}{\pi} .
$$

If $[m \alpha / \pi]<m \alpha / \pi$, then, whenever $-\tau>0$ is small enough,

$$
\alpha+\tau=\frac{2 t+\chi}{2 m} \pi, \quad \text { with } t=\left[\frac{m \alpha}{\pi}\right] \geq 0,0<\chi=2\left(\frac{m \alpha}{\pi}-\left[\frac{m \alpha}{\pi}\right]\right)+\frac{2 m \tau}{\pi}<2 .
$$

Especially in this case, when $m \geq 2$ we obtain $t=[m \alpha / \pi] \geq[2 \alpha / \pi]>1$.

Thus for any integer $m \geq 2$, if $-\tau>0$ is small enough, then

$$
\alpha+\tau=\frac{2 t+\chi}{2 m} \pi, \quad \text { with } t \geq 1,0<\chi<2 .
$$

When $m=1$, by (6.19) we obtain that if $-\tau>0$ is small enough, then

$$
\alpha+\tau=\frac{2 t+\chi}{2} \pi, \quad \text { with } t=0,0<\chi=\frac{2}{\pi}(\alpha+\tau)<2 .
$$

$2^{\circ}$. By (6.16), we can choose $\tau>0$ so small that

$$
\frac{b}{2} \sin (2 \tau)+\left(1-\frac{b^{2}}{2}\right) \sin ^{2} \tau<0
$$

to perturb the eigenvalue -1 of $M_{1}$ to nearby negative eigenvalues $\lambda^{ \pm 1} \in \mathbf{R} \backslash\{-1\}$.

Therefore in the case of $k=1$, we have proved that $M_{1} R(\tau)$ perturbs the eigenvalue -1 of $M_{1}$ to nearby eigenvalues on the unit circle by choosing small $\tau \neq 0$ 
suitably, and that $M_{1} R(\tau)$ or $M_{1} D\left(2^{\tau}\right)$ perturbs the eigenvalue -1 of $M_{1}$ to nearby eigenvalues on $\mathbf{R} \backslash\{-1\}$ by choosing small $\tau \neq 0$ suitably.

Combining Theorem 6.1 and the above discussions on perturbations, by induction we have proved the following main result of this section.

Theorem 6.2. Suppose $M \in \operatorname{Sp}(2 n)$ possesses the eigenvalue -1 . Then there exist a matrix $P \in \operatorname{Sp}(2 n)$ and a perturbation matrix $G(\tau)=G_{1}\left(\tau_{1}\right) \diamond \cdots \diamond G_{p}\left(\tau_{p}\right) \diamond I$ such that $P^{-1} M P$ is a $\diamond$-product given by (6.6), each $G_{i}\left(\tau_{i}\right)$ with $\tau_{i} \neq 0$ is determined in the above discussion according to $M_{i}$ in (6.6) for $1 \leq i \leq p$, and the eigenvalue -1 of $M$ is perturbed away from itself to nearby eigenvalues of $P^{-1} M P G(\tau)$ on $\mathbf{U} \backslash \mathbf{R}$, or on $\mathbf{R} \backslash\{-1\}$, while all the other eigenvalues of $M$ remain fixed.

Based upon Theorem 6.1, we can give the following definition concerning symplectic matrices possessing eigenvalue -1 .

Definition 6.3. Suppose $M \in \operatorname{Sp}(2 n)$ and $-1 \in \sigma(M)$. Denote the set of all normal forms of eigenvalue -1 appearing in the $\diamond$-decomposition (6.6) of $M$ by $\mathcal{J}_{-1}(M)$. We define

(6.22) $\omega_{2}(M)=\operatorname{dim} \operatorname{span}\left\{\right.$ eigenvectors of $\left.M_{i} \mid M_{i} \in \mathcal{J}_{-1}(M) \cap \operatorname{Sp}\left(2 k_{i}\right), k_{i} \geq 2\right\}$,

(6.23) $\omega_{+}(M)=\#\left\{M_{i} \in \mathcal{J}_{-1}(M) \mid M_{i}=\left(\begin{array}{cc}-1 & 1 \\ 0 & -1\end{array}\right)\right\}$,

(6.24) $\omega_{0}(M)=\#\left\{M_{i} \in \mathcal{J}_{-1}(M) \mid M_{i}=\left(\begin{array}{cc}-1 & 0 \\ 0 & -1\end{array}\right)\right\}$,

(6.25) $\omega_{-}(M)=\#\left\{M_{i} \in \mathcal{J}_{-1}(M) \mid M_{i}=\left(\begin{array}{cc}-1 & -1 \\ 0 & -1\end{array}\right)\right\}$,

and

$$
\omega(M)=\omega_{2}(M)+\omega_{-}(M) .
$$

Then we always have

$$
\omega(M) \leq \min \left\{n, \frac{1}{2} \operatorname{dim} E_{-1}\right\}, \quad \forall M \in \operatorname{Sp}(2 n) .
$$

\section{Perturbations of eigenvalues AWAY FROM ROOTS OF UNITY $e^{ \pm \theta \sqrt{-1}} \notin \mathbf{R}$}

In [LD] the following two basic normal forms of symplectic matrices with eigenvalues $\lambda \in \mathbf{U} \backslash \mathbf{R}$ are introduced.

Normal form $1 . M_{1} \in \mathrm{Sp}(4 k)$ with some integer $k \geq 1$ defined by

$$
M_{1}=\left(\begin{array}{cc}
A & B \\
0 & C
\end{array}\right)
$$

where $A$ is a $2 k \times 2 k$ Jordan block form matrix of eigenvalue $\lambda=e^{\theta \sqrt{-1}}$ :

$$
A=\left(\begin{array}{cccccc}
R(\theta) & I_{2} & 0 & \ldots & 0 & 0 \\
0 & R(\theta) & I_{2} & \ldots & 0 & 0 \\
0 & 0 & R(\theta) & \ldots & 0 & 0 \\
\cdot & . & \cdot & \ldots & . & . \\
\cdot & . & . & \ldots & . & . \\
0 & 0 & 0 & \ldots & R(\theta) & I_{2} \\
0 & 0 & 0 & \ldots & 0 & R(\theta)
\end{array}\right),
$$


$C$ is a $2 k \times 2 k$ matrix of $2 \times 2$ blockwise lower triangular matrix of the form

$$
C=\left(\begin{array}{ccccc}
R(\theta) & 0 & \ldots & 0 & 0 \\
-R(2 \theta) & R(\theta) & \ldots & 0 & 0 \\
\cdot & \cdot & \ldots & \cdot & \cdot \\
\cdot & \cdot & \ldots & \cdot & \cdot \\
(-1)^{k} R((k-1) \theta) & (-1)^{k-1} R((k-2) \theta) & \ldots & R(\theta) & 0 \\
(-1)^{k+1} R(k \theta) & (-1)^{k} R((k-1) \theta) & \ldots & -R(2 \theta) & R(\theta)
\end{array}\right)
$$

and $B$ is a $2 k \times 2 k$ matrix formed by $2 \times 2$ matrices $B_{i, j}$ :

$$
B=\left(B_{i, j}\right)_{1 \leq i, j \leq k}, \quad B_{i, j}=0 \quad \text { if } j>i+1 .
$$

Normal form 2. $M_{1} \in \operatorname{Sp}(4 k+2)$ with some integer $k \geq 0$ defined by

$$
M_{1}=\left(\begin{array}{cccc}
A & D & B & E \\
0 & \cos \hat{\theta} & F^{T} & -\sin \hat{\theta} \\
0 & 0 & C & 0 \\
0 & \sin \hat{\theta} & G^{T} & \cos \hat{\theta}
\end{array}\right) .
$$

where $A, B$, and $C$ are $2 k \times 2 k$ matrices, $D, E, F$, and $G$ are $2 k \times 1$ matrices, $A$ is given by (7.2), $C$ is given by (7.3), and

$$
\hat{\theta}=\theta, \quad D=(0, \ldots, 0,1,0)^{T}, \quad E=(0, \ldots, 0,0,1)^{T},
$$

or

$$
\hat{\theta}=-\theta, \quad D=(0, \ldots, 0,0,1)^{T}, \quad E=(0, \ldots, 0,1,0)^{T} .
$$

In these two normal forms, $M_{1}$ possesses either two or four linearly independent eigenvectors of $\lambda^{ \pm 1}$.

In $[\mathrm{LD}]$ the following result on the normal forms of symplectic matrices with the eigenvalue $\lambda \in \mathbf{U} \backslash \mathbf{R}$ is proved.

Theorem 7.1. Suppose $M \in \operatorname{Sp}(2 n)$ possesses the eigenvalue $\alpha^{ \pm 1} \in \mathbf{U} \backslash \mathbf{R}$. Then there exist $P \in \operatorname{Sp}(2 n)$ and $p \in \mathbf{N}$ such that

$$
P^{-1} M P=M_{1} \diamond \cdots \diamond M_{p} \diamond M_{0},
$$

where $M_{0} \in \operatorname{Sp}\left(2 k_{0}\right)$ with $k_{0} \geq 0$ and $\lambda \notin \sigma\left(M_{0}\right), k_{i} \geq 1$, and $M_{i} \in \operatorname{Sp}\left(2 k_{i}\right)$ is of the normal form 1 or 2 defined above for $1 \leq i \leq p$. Let $E_{\lambda}$ denote the invariant root vector space of $M$ belonging to the eigenvalue $\lambda^{ \pm 1}$. Then $2 \sum_{i=0}^{m} k_{i}=$ $2 k_{0}+\operatorname{dim} E_{\lambda}=2 n$.

Now we suppose $M \in \operatorname{Sp}(2 n)$ and $\lambda^{ \pm 1} \equiv e^{ \pm \theta \sqrt{-1}} \in(\mathbf{U} \backslash \mathbf{R}) \cap \sigma(M)$ are $m$ th roots of unity for some integer $m \geq 2$. Based upon the normal form Theorem 7.1, we study the perturbations of $M$ so that the eigenvalues $\lambda^{ \pm 1}$ are perturbed to nearby eigenvalues on the unit circle.

Since the eigenvalues $\lambda^{ \pm 1}=e^{ \pm \theta \sqrt{-1}} \in \mathbf{U} \backslash \mathbf{R}$ are $m$ th roots of unity, we suppose $\theta=2 t \pi / m$ for some integer $t \in[1, m-1] \backslash\{m / 2\}$. We start from the two normal form cases.

Case 1. Perturbations on the normal form 1.

We multiply the matrix $M_{1}$ by the $4 k \times 4 k$ matrix

$$
G_{1}(\tau) \equiv \operatorname{diag}\left(R\left(\frac{\tau}{k}\right), R\left(\frac{2 \tau}{k}\right), \ldots, R(\tau), R\left(\frac{\tau}{k}\right), R\left(\frac{2 \tau}{k}\right), \ldots, R(\tau)\right),
$$


with $\tau>0$ mall. When $\tau>0$ is small enough and suitably chosen, we find that the $2 k$-multiple eigenvalue $e^{ \pm \theta \sqrt{-1}}$ of $M_{1}$ is perturbed to $k$ double eigenvalues

$$
\alpha_{i}^{ \pm 1}=e^{ \pm\left(\theta+\frac{i}{k} \tau\right) \sqrt{-1}} \quad \text { for } i=1, \ldots, k
$$

of the matrix $M_{1} G_{1}(\tau)$, and these $\alpha_{i}^{ \pm 1}$,s are not $m$ th roots of unity.

For $u=\left(u_{1}, \ldots, u_{2 k}\right)$ and $v=\left(v_{1}, \ldots, v_{2 k}\right)$ with $0<u_{1}<\cdots<u_{2 k}$ and $|v|$ sufficiently small, we define

$$
G(\tau, u, v)=G_{1}(\tau)\left[\left(D\left(2^{v_{1}}\right) R\left(u_{1}\right)\right) \diamond \cdots \diamond\left(D\left(2^{v_{2 k}}\right) R\left(u_{2 k}\right)\right)\right] .
$$

Then by suitably choosing small enough $u$ and small $v$, we obtain that the eigenvalues in (7.10) are perturbed to $4 k$ different simple eigenvalues of $M_{1} G(\tau, u, v)$ on the unit circle, and these eigenvalues can be listed as

$$
\exp \left( \pm\left(\theta+\frac{i}{k} \tau+\varepsilon_{2 i-1}\right) \sqrt{-1}\right) \quad \text { and } \exp \left( \pm\left(\theta+\frac{i}{k} \tau+\varepsilon_{2 i}\right) \sqrt{-1}\right)
$$

for $1 \leq i \leq k$, with some sufficiently small pairwise different constants $\varepsilon_{1}, \ldots, \varepsilon_{2 k}$.

Since $\theta=2 t \pi / m$ for some integer $t \in[1, m-1] \backslash\{m / 2\}$, for $1 \leq i \leq k$ and $j=2 i-1$ or $2 i$ we obtain

$$
\theta+\frac{i}{k} \tau+\varepsilon_{j}=\frac{2 t_{j}+\chi_{j}}{m} \pi, \quad \text { with } t_{j} \geq t \geq 1 \text { and } 0<\chi_{j}<2 .
$$

Note that in this case the matrix $M_{1}$ has at most 4 eigenvectors, the matrix $M_{1} G(\tau, u, v)$ has $4 k$ eigenvectors, and

$$
2 \sum_{j=1}^{2 k} t_{j} \geq 4 k \geq 4 \geq \operatorname{dim} \operatorname{span}\left\{\text { eigenvectors of } M_{1} \text { belonging to } \lambda^{ \pm 1}\right\} .
$$

Thus we have proved that there exists a $4 k \times 4 k$ rotation matrix $G(\tau, u, v)$, with both $\tau>0$ and $|u|$ small enough, such that by this rotational perturbation the eigenvalues $e^{ \pm \theta \sqrt{-1}}$ of $M$ are changed to $k$ simple eigenvalues given by (7.12) of $M_{1} G(\tau, u, v)$. These new simple eigenvalues belong to $\mathbf{U} \backslash \mathbf{R}$, are not $m$ th roots of unity, and the corresponding normal forms in the sense of Definition 3.5 satisfy (7.13) and (7.14).

Case 2. Perturbations on the normal form 2.

Similarly to the discussion of Case 1 , there is a $(4 k+2) \times(4 k+2)$ rotation matrix

$$
\begin{gathered}
G_{1}(\tau) \equiv \operatorname{diag}\left(R\left(\frac{\tau}{k}\right), R\left(\frac{2 \tau}{k}\right), \ldots, R(\tau), R\left(\frac{\tau}{k}\right), R\left(\frac{2 \tau}{k}\right), \ldots, R(\tau)\right) \diamond R(\tau), \\
G(\tau, u, v)=G_{1}(\tau)\left[\left(D\left(2^{v_{1}}\right) R\left(u_{1}\right)\right) \diamond \cdots \diamond\left(D\left(2^{v_{2 k}}\right) R\left(u_{2 k}\right)\right) \diamond I_{2}\right],
\end{gathered}
$$

with $u=\left(u_{1}, \ldots, u_{2 k}\right), 0<u_{1}<\cdots<u_{2 k}$ and $0<\tau$ small enough, such that by this rotational perturbation the eigenvalues $\lambda^{ \pm 1}=e^{ \pm \theta \sqrt{-1}}$ of $M_{1}$ are changed to $4 k+2$ nearby simple eigenvalues of $M_{1} G(\tau, u)$ given by $(7.12)$ and

$$
\exp ( \pm(\hat{\theta}+\tau) \sqrt{-1})
$$


These new simple eigenvalues belong to $\mathbf{U} \backslash \mathbf{R}$, are not $m$ th roots of unity, and the corresponding normal forms in the sense of Definition 3.5 satisfy relations (7.13) and

$$
\hat{\theta}+\tau=\frac{2 \hat{t}+\hat{\chi}}{m} \pi, \quad \text { with } \hat{t} \geq t \geq 1,0<\hat{\chi}<2,
$$

$$
2 \sum_{j=1}^{2 k} t_{j}+2 \hat{t} \geq 4 k+2 \geq \operatorname{dim} \operatorname{span}\left\{\text { eigenvectors of } M_{1} \text { belonging to } \lambda^{ \pm 1}\right\}
$$

The discussions of the two basic normal forms are complete.

Combining the discussions of these two cases, by induction, we have proved the following main result of this section.

Theorem 7.2. Suppose $M \in \operatorname{Sp}(2 n)$ and $\lambda^{ \pm 1} \equiv e^{ \pm \theta \sqrt{-1}} \in(\mathbf{U} \backslash \mathbf{R}) \cap \sigma(M)$ are roots of unity. Then there exist a matrix $P \in \mathrm{Sp}(2 n)$ and a perturbation matrix $G(\tau, u, v)$ which is a $\diamond$-product of matrices defined by (7.11) and (7.15) depending on small parameters $\tau>0, u$, and $v$ such that $P^{-1} M P$ is a $\diamond$-product given by (7.8) in Theorem 7.1, and by the perturbation the eigenvalues $\lambda^{ \pm 1}$ of $M$ are changed to nearby simple eigenvalues of $P^{-1} M P(G(\tau, u, v) \diamond I)$ given by (7.12) or (7.12) and (7.16). These new simple eigenvalues belong to $\mathbf{U} \backslash \mathbf{R}$, are not mth roots of unity, and corresponding normal forms in the sense of Definition 3.5 satisfy relations (7.13) and (7.14) or (7.13), (7.17) and (7.18). All the other eigenvalues of $M$ are fixed in the perturbation.

For later convenience, based upon Theorem 7.1, we give the following definition.

Definition 7.3. For $k \in \mathbf{N}$, suppose $M \in \operatorname{Sp}(2 n)$ possesses eigenvalues $\lambda_{i}^{ \pm}=$ $e^{ \pm \theta_{i} \sqrt{-1}} \in \mathbf{U} \backslash \mathbf{R}, 1 \leq i \leq r$, which are $k$ th roots of unity. Denote the set of all normal forms in the $\diamond$-decomposition (7.8) of $M$ by $\mathcal{J}_{\lambda}(M)$. We define

$$
\begin{gathered}
\varphi_{k, 2}(M)=\#\left\{M_{i} \mid M_{i} \in \mathcal{J}_{\lambda}(M) \text { for some } \lambda^{ \pm 1} \in \sigma(M) \cap(\mathbf{U} \backslash \mathbf{R}),\left(\lambda^{ \pm 1}\right)^{k}=1,\right. \\
\left.M_{i} \text { possesses } 2 \text { independent eigenvectors of } \lambda^{ \pm 1}\right\},
\end{gathered}
$$

$$
\begin{gathered}
\varphi_{k, 4}(M)=\#\left\{M_{i} \mid M_{i} \in \mathcal{J}_{\lambda}(M) \text { for some } \lambda^{ \pm 1} \in \sigma(M) \cap(\mathbf{U} \backslash \mathbf{R}),\left(\lambda^{ \pm 1}\right)^{k}=1,\right. \\
\left.M_{i} \text { possesses } 4 \text { independent eigenvectors of } \lambda^{ \pm 1}\right\} .
\end{gathered}
$$

Define

$$
\varphi_{k}(M)=\varphi_{k, 2}(M)+2 \varphi_{K, 4}(M) .
$$

Then we always have

$$
\varphi_{k}(M) \leq n \quad \forall M \in \operatorname{Sp}(2 n)
$$

Note that $2 \varphi_{k}(M)$ is the total number of all real eigenvectors belonging to any eigenvalue of $M$ which is a nonreal $k$ th root of unity. 


\section{The iteration FORMUla FOR DEGENERATE LINEAR HAMILTONIAN SYSTEMS}

In this section, we consider the linear Hamiltonian system

$$
\dot{y}=J B(t) y,
$$

where $B \in C\left(S_{T}, \mathcal{L}_{s}\left(\mathbf{R}^{2 n}\right)\right)$. As in $\S 4$, we denote the fundamental solution of (8.1) defined on $[0,1]$ by $\gamma:[0,1] \rightarrow \operatorname{Sp}(2 n)$ with $\gamma(0)=I$. Denote the Maslov-type index of $(8.1)$ by $\left(i_{T}, \nu_{T}\right)=\left(i_{T}(\gamma), \nu_{T}(\gamma)\right)$. For $k \in \mathbf{N}$, when $B$ is viewed as defined on $S_{k T}$, the path $\tilde{\gamma}$ defined by $(4.2)$ is the fundamental solution of (8.1) on $[0, k]$. We denote the corresponding Maslov-type index of (8.1) defined on $[0, k]$ by $\left(i_{k T}, \nu_{k T}\right)=\left(i_{k T}(\tilde{\gamma}), \nu_{k T}(\tilde{\gamma})\right)$.

Lemma 8.1. For $k \in \mathbf{N}$

$$
\nu_{k T}=2 \varphi_{k}+\nu_{T}+\left(\frac{1+(-1)^{k}}{2}\right)\left(\omega+\omega_{+}+2 \omega_{0}\right),
$$

where $\omega=\omega(\gamma(T)), \omega_{+}=\omega_{+}(\gamma(T)), \omega_{0}=\omega_{0}(\gamma(T))$, and $\varphi_{k}=\varphi_{k}(\gamma(T))$ are given by Definitions 6.3 and 7.3 .

Proof. For any $m \times m$ real matrix $M$ and any $k \in \mathbf{N}$, by using the Jordan normal form of $M$ on the field $\mathbf{C}$ one can prove that

$$
\begin{aligned}
& \operatorname{dim} \operatorname{ker}\left(M^{k}-I\right) \\
& \quad=\operatorname{dim} \operatorname{span}\left\{\xi \in \mathbf{C}^{m} \mid \exists \lambda \in \mathbf{C} \text { such that } \lambda^{k}=1, M \xi=\lambda \xi\right\} .
\end{aligned}
$$

This implies (8.2).

Lemma 8.2. Under the above assumptions, for any $P \in \operatorname{Sp}(2 n)$ and $k \in \mathbf{N}$

$$
\begin{array}{cc}
i_{T}(\beta)=i_{T}(\gamma), & \nu_{T}(\beta)=\nu_{T}(\gamma), \\
i_{k T}(\tilde{\beta})=i_{k T}(\tilde{\gamma}), & \nu_{k T}(\tilde{\beta})=\nu_{k T}(\tilde{\gamma}),
\end{array}
$$

where $\beta(t)=P^{-1} \gamma(t) P$ for $0 \leq t \leq T$.

Proof. By Corollary 2.9, we obtain (8.3). Since

$$
\tilde{\beta}(k T)=\beta(T)^{k}=\left(P^{-1} \gamma(T) P\right)^{k}=P^{-1} \gamma(T)^{k} P
$$

we obtain

$$
\nu_{k T}(\tilde{\beta})=\operatorname{dim} \operatorname{ker}\left(P^{-1} \gamma(T)^{k} P-I\right)=\operatorname{dim} \operatorname{ker}\left(\gamma(T)^{k}-I\right)=\nu_{k T}(\tilde{\gamma}) .
$$

So the second equality in (8.4) holds. Viewing $B$ as defined on $S_{k T}$, by the second equality in (8.4), the same argument in the proof of Corollary 2.9 yields the first equality in (8.4), and completes the proof.

The main result in this section is the following theorem:

Theorem 8.3. Suppose $\nu_{k T}>0$ for $k \in \mathbf{N}$, i.e. $\gamma(T) \in \operatorname{Sp}(2 n)_{k}^{0}$. Then there exist smooth perturbation paths $\gamma_{ \pm 1}:[0, T] \rightarrow \operatorname{Sp}(2 n)$ with $\gamma_{ \pm 1}(0)=I$ and $\gamma_{ \pm 1}(T) \in$ $\mathrm{Sp}(2 n)_{k}^{*}$ such that

$$
i_{T}\left(\gamma_{-1}\right)=i_{T}(\gamma)=i_{T}\left(\gamma_{1}\right)-\nu_{T}(\gamma)
$$

We can further choose the perturbation paths $\gamma_{ \pm 1}$ so that there exist integers $\mu^{ \pm}$ with $0 \leq \varphi_{k} \leq \mu^{ \pm} \leq n$, and integers $t_{j}^{ \pm}, 0 \leq t_{j}^{ \pm} \leq k-1$ for $1 \leq j \leq \mu^{ \pm}$, which are 
determined by the normal form in the same path-connected component of $\operatorname{Sp}(2 n)_{k}^{*}$ with $\gamma_{ \pm 1}(T)$ such that

$1^{\circ}$. the following inequality holds:

$$
\begin{gathered}
k\left(i_{T}-\mu^{-}\right)+\sum_{j=1}^{\mu^{-}} 2 t_{j}^{-}+\mu^{-}=i_{k T}\left(\tilde{\gamma}_{-1}\right) \geq i_{k T} \geq i_{k T}\left(\tilde{\gamma}_{1}\right)-\nu_{k T} \\
=k\left(i_{T}+\nu_{T}-\mu^{+}\right)+\sum_{j=1}^{\mu^{+}} 2 t_{j}^{+}+\mu^{+}-\nu_{k T}
\end{gathered}
$$

$2^{\circ}$. if $\mu^{+}=n$, then the integers $\mu^{+}, i_{T}+\nu_{T}$, and $i_{k T}\left(\tilde{\gamma}_{1}\right)$ possess the same parity; $3^{\circ}$. if $\mu^{-}=n$, then the integers $\mu^{-}, i_{T}$, and $i_{k T}\left(\tilde{\gamma}_{-1}\right)$ possess the same parity; $4^{\circ}$. the inequality

$$
\sum_{j=1}^{\mu^{+}} 2 t_{j}^{+} \geq \nu_{k T}-\nu_{T}-\frac{\left(1+(-1)^{k}\right)}{2} \omega
$$

holds, where $\omega=\omega(\gamma(T))$ is given by Definition 6.3.

Proof. Without loss of generality, we may assume $T=1$. We carry out the proof in several steps.

Step 1. If $1 \in \sigma(\gamma(1))$, denote the root vector space belonging to the eigenvalue 1 of $\gamma(1)$ by $E_{1}$. By $\S 5$ there exist a matrix $P_{1} \in \operatorname{Sp}(2 n)$ and a one-parameter family of perturbations

$$
G_{1}(\theta)=R_{h_{1}}(\theta) \diamond \cdots \diamond R_{h_{q}}(\theta)
$$

such that $P_{1}^{-1} \gamma(1) P_{1} G_{1}(\theta)=P_{1}^{-1} \gamma(1) P_{1}$ on $P_{1}^{-1} E_{1}^{\perp}$, and $1 \notin \sigma\left(P_{1}^{-1} \gamma(1) P_{1} G_{1}(\theta)\right)$ with $0<|\theta| \leq \theta_{1}$ for some $\theta_{1}>0$ sufficiently small.

As in $\S 2$ (cf. [Lo1, Lo7]), we define an increasing function $\rho \in C^{\infty}(\mathbf{R},[0,1])$ such that $\rho(t)=0$ for $t \leq 0$ and $\rho(t)=1$ for $t \geq 1$. Then we define the perturbation paths $\alpha_{s}:[0,1] \rightarrow \operatorname{Sp}(2 n)$ for $-1 \leq s \leq 1$ by

$$
\alpha_{s}(t)=P_{1}^{-1} \gamma(t) P_{1} G_{1}\left(s \rho(t) \theta_{1}\right) \quad \text { for } 0 \leq t \leq 1 \text {. }
$$

Note that $\alpha_{0}=\gamma$ and $\alpha_{s}(1) \in \operatorname{Sp}(2 n)^{*}$ for $s \neq 0$. By equations in (8.3) and (8.4) of Lemma 8.2, the paths $P_{1}^{-1} \gamma P_{1}$ and $\gamma$ possess the same Maslov-type indices. Thus by the definition of Maslov-type index given in $\S 2$ (cf. [Lo1, Lo7]) we obtain

$$
\begin{gathered}
\nu_{1}\left(\alpha_{-s}\right)=\nu_{1}\left(\alpha_{s}\right)=0, \\
i_{1}\left(\alpha_{-s}\right)=i_{1}(\gamma)=i_{1}\left(\alpha_{s}\right)-\nu_{1}(\gamma),
\end{gathered}
$$

for $0<s \leq 1$ and small $\theta_{1}>0$. We further require $\theta_{1}>0$ to be small enough so that the eigenvalue 1 of $\gamma(1)$ is perturbed to some new eigenvalue of $\alpha_{s}(1)$, which is not a $k$ th root of unity, for $s \in[-1,1] \backslash\{0\}$.

Step 2. If $-1 \in \sigma(\gamma(1))$ and $k \in 2 \mathbf{N}$, then $-1 \in \sigma\left(\alpha_{s}(1)\right)$ for $s \in[-1,1]$. Denote the root vector space belonging to the eigenvalue -1 of $\alpha_{1}(1)$ by $E_{-1}$. By $\S 6$, there exist a matrix $P_{2} \in \operatorname{Sp}(2 n)$ and a one-parameter family of perturbations $G_{2}(\theta)$ which is a $\diamond$-product of matrices of the forms

$$
\operatorname{diag}(R(\theta), \ldots, R(\theta)) \quad \text { or } \quad \operatorname{diag}(R(\theta), \ldots, R(\theta)) \diamond R\left(\theta^{ \pm}\right)
$$


with $\theta^{ \pm}=\theta$ or $\theta^{ \pm}=-\theta$, such that $P_{2}^{-1} \alpha_{1}(1) P_{2} G_{2}(\theta)=P_{2}^{-1} \alpha_{1}(1) P_{2}$ on $P_{2}^{-1} E_{-1}^{\perp}$, and $-1 \notin \sigma\left(P_{2}^{-1} \alpha_{1}(1) P_{2} G_{2}(\theta)\right)$ with $0<|\theta| \leq \theta_{2}$ for some $\theta_{2}>0$ sufficiently small.

As in Step 1, we define the perturbation paths $\beta_{s}:[0,1] \rightarrow \operatorname{Sp}(2 n)$ for $0 \leq s \leq 1$ by

$$
\beta_{s}(t)=P_{2}^{-1} \alpha_{1}(t) P_{2} G_{2}\left(s \rho(t) \theta_{2}\right) \quad \text { for } 0 \leq t \leq 1 \text {. }
$$

Note that $\beta_{0}=P_{2}^{-1} \alpha_{1} P_{2}$. Since $\alpha_{1}$ is a nondegenerate path in $\operatorname{Sp}(2 n)$, if $\theta_{2}>0$ is small, for $0 \leq s \leq 1$ we have

$$
\begin{gathered}
\nu_{1}\left(\beta_{s}\right)=\nu_{1}\left(\alpha_{1}\right)=0, \\
i_{1}\left(\beta_{s}\right)=i_{1}\left(\alpha_{1}\right) .
\end{gathered}
$$

By $\S 6$, we can choose the perturbations and further require $\theta_{2}>0$ to be small enough so that when $s \neq 0$, the eigenvalue -1 of $\alpha_{1}(1)$ is perturbed to eigenvalues of $\beta_{s}(1)$ which are completely on the unit circle but not $k$ th roots of unity, or which are completely on $\mathbf{R} \backslash\{-1\}$.

Step 3. If $\lambda^{ \pm 1} \equiv e^{ \pm \theta \sqrt{-1}} \in \sigma(\gamma(1)) \cap(\mathbf{U} \backslash \mathbf{R})$ are $k$ th roots of unity, then $\lambda^{ \pm 1} \in$ $\sigma\left(\beta_{s}(1)\right)$ for $s \in[-1,1]$. Denote the root vector space belonging to the eigenvalue $\lambda^{ \pm 1}$ of $\beta_{1}(1)$ by $E_{\lambda}$. By $\S 7$ there exist a matrix $P_{3} \in \operatorname{Sp}(2 n)$ and a one-parameter family of perturbation matrices $G_{3}(\theta)$ which is a $\diamond$-product of matrices of the forms (7.11) or (7.15), such that $P_{3}^{-1} \beta_{1}(1) P_{3} G_{3}(\theta)=P_{3}^{-1} \beta_{1}(1) P_{3}$ on $P_{3}^{-1} E_{\lambda}^{\perp}$, and $\lambda^{ \pm 1} \notin$ $\sigma\left(P_{3}^{-1} \beta_{1}(1) P_{3} G_{3}(\theta)\right)$ with $0<\theta \leq \theta_{3}$ for some $\theta_{3}>0$ sufficiently small. by

As in Step 2, we define the perturbation paths $\gamma_{s}:[0,1] \rightarrow \operatorname{Sp}(2 n)$ for $0 \leq s \leq 1$

$$
\gamma_{s}(t)=P_{3}^{-1} \beta_{1}(t) P_{3} G_{3}\left(s \rho(t) \theta_{3}\right) \quad \text { for } 0 \leq t \leq 1
$$

Note that $\gamma_{0}=P_{3}^{-1} \beta_{1} P_{3}$. Since $\beta_{1}$ is a nondegenerate path in $\operatorname{Sp}(2 n)$, if $\theta_{3}>0$ is small enough, for $0 \leq s \leq 1$ we have

$$
\begin{gathered}
\nu_{1}\left(\gamma_{s}\right)=\nu_{1}\left(\beta_{1}\right)=0, \\
i_{1}\left(\gamma_{s}\right)=i_{1}\left(\beta_{1}\right) .
\end{gathered}
$$

By $\S 7$, we can further require $\theta_{3}>0$ to be small enough so that when $s \neq 0$ the eigenvalue $\lambda^{ \pm 1}$ of $\beta_{1}(1)$ is perturbed to eigenvalues of $\gamma_{s}(1)$ which are on the unit circle but are not $k$ th roots of unity.

Repeating this procedure, we can perturb all the eigenvalues of $\beta_{1}(1)$ which are nonreal $k$ th roots of unity away from themselves to nearby suitable values on the unit circle as in $\S 7$. We still use $\gamma_{1}$ to denote the final perturbation path.

Note that starting from Step 2, the same procedure also works for the paths $\alpha_{-1}$ and $\beta_{-1}$, and we use $\gamma_{-1}$ to denote the final perturbation path. These paths satisfy

$$
\begin{gathered}
\nu_{1}\left(\gamma_{ \pm 1}\right)=0 \\
i_{1}\left(\gamma_{-1}\right)=i_{1}(\gamma)=i_{1}\left(\gamma_{1}\right)-\nu_{1}(\gamma) \\
\nu_{k}\left(\tilde{\gamma}_{ \pm 1}\right)=0 .
\end{gathered}
$$


Step 4. Let $B_{ \pm}(t)=-J \dot{\gamma}_{ \pm 1}(t) \gamma_{ \pm 1}^{-1}(t)$ for $t \in[0,1]$. The smooth perturbation paths $\gamma_{ \pm 1}$ we obtained above possess the following properties:

(1) They can be chosen to be as close to the given path $\gamma$ as we want by requiring $\max \left\{\theta_{i} \mid i=1,2,3\right\}>0$ to be small enough.

(2) $B_{ \pm} \in C\left(S_{1}, \mathcal{L}_{s}\left(\mathbf{R}^{2 n}\right)\right)$.

(3) They satisfy (8.19)-(8.21).

Step 5. By Theorem 4.1 and (3) of Step 4, from the normal forms in the same path-connected component of $\operatorname{Sp}(2 n)_{k}^{*}$ with $\gamma_{ \pm 1}(1)$, there exist integers $\mu^{ \pm}$with $0 \leq \mu^{ \pm} \leq n$ and integers $t_{j}^{ \pm}$with $0 \leq t_{j}^{ \pm} \leq k-1$ for $1 \leq j \leq \mu^{ \pm}$, such that

$$
i_{k}\left(\tilde{\gamma}_{ \pm 1}\right)=k\left(i_{1}\left(\gamma_{ \pm 1}\right)-\mu^{ \pm}\right)+\sum_{j=1}^{\mu^{ \pm}} 2 t_{j}^{ \pm}+\mu^{ \pm} .
$$

As in the proof of Lemma 8.2, by the saddle point reduction method of [AZ] we obtain a finite-dimensional subspace $Z$ of $E=L^{2}\left(S_{k}, \mathbf{R}^{2 n}\right)$ with $\operatorname{dim} Z=2 d$ for some large integer $d>0$ and three injective maps $u$ and $u_{ \pm}: Z \rightarrow \operatorname{dom}(A)$ with $A=-J \frac{d}{d t}$, such that the Morse indices $m^{+}, m^{0}, m^{-}$, and $m_{ \pm}^{+}, m_{ \pm}^{0}, m_{ \pm}^{-}$of the functionals

$$
\begin{aligned}
a(z) & =\frac{1}{2}\langle(A-B) u(z), u(z)\rangle_{L^{2}}, \quad \forall z \in Z, \\
a_{ \pm}(z) & =\frac{1}{2}\left\langle\left(A-B_{ \pm}\right) u_{ \pm}(z), u_{ \pm}(z)\right\rangle_{L^{2}}, \quad \forall z \in Z,
\end{aligned}
$$

at the origin satisfy the following equations:

$$
\begin{aligned}
& m^{+}=d-i_{k}(\tilde{\gamma})-\nu_{k}(\tilde{\gamma}), \quad m^{0}=\nu_{k}(\tilde{\gamma}), \quad m^{-}=d+i_{k}(\gamma), \\
& m_{ \pm}^{+}=d-i_{k}\left(\tilde{\gamma}_{ \pm 1}\right), \quad m_{ \pm}^{0}=0, \quad m_{ \pm}^{-}=d+i_{k}\left(\tilde{\gamma}_{ \pm 1}\right),
\end{aligned}
$$

where we have used (8.21).

By (1) of Step 4, we obtain

$$
m_{-}^{-} \geq m^{-} \geq m_{+}^{-}-m^{0} .
$$

Combining this with (8.23), (8.24) and (3) of Step 4 yields

$$
i_{k}\left(\tilde{\gamma}_{-1}\right) \geq i_{k}(\tilde{\gamma}) \geq i_{k}\left(\tilde{\gamma}_{1}\right)-\nu_{k}(\tilde{\gamma}) .
$$

Combining this with (8.19)-(8.22) yields (8.8), and proves $1^{\circ}$ of Theorem 8.3.

Note that $2^{\circ}$ and $3^{\circ}$ are direct consequences of Theorem 4.1 .

Step 6. The proof of $4^{\circ}$ of Theorem 8.3.

Note that $\nu_{k} \equiv \nu_{k}(\tilde{\gamma})$ is the total geometric multiplicity of eigenvalues of $\gamma(1)$ which are $k$ th roots of unity. Set $\varphi_{k}=\varphi_{k}(\gamma(1))$ (given by Definition 7.3).

Now we further require that the path $\gamma_{1}$ be obtained specifically in the following way:

$1^{\circ}$. If $\lambda^{ \pm 1} \equiv e^{ \pm \theta \sqrt{-1}} \in \sigma(\gamma(1))$ is a nonreal $k$ th root of unity, then $\theta=2 t \pi / k$ with $1 \leq t \leq k-1$ and $t \neq k / 2$. Thus, by $\S 7$, the perturbation we made for small $\tau>0$ in Step 2 yields that the eigenvalues $e^{ \pm \theta \sqrt{-1}}$ of $\gamma(1)$ are perturbed to simple eigenvalues of $\gamma_{1}(1)$, which are of the forms (7.12) or (7.12) and (7.16). As in $\S 7$, we next use the discussion of (7.13), (7.14), and (7.17), (7.18). If $M_{i}$ is a $2 \times 2$ normal Jordan block belonging to $\lambda^{ \pm 1}$ of $\gamma(1)$, then the perturbation on $M_{i}$ defined 
in Step 2 produces a $2 \times 2$ rotational matrix $R\left(\theta_{i}\right)$ in the normal form of the $k$ th nondegenerate matrix $\gamma_{1}(1)$ as defined in (3.3) or (3.4) such that

$$
\theta_{i}=\frac{2 t_{i}+\chi_{i}}{k} \pi, \quad 1 \leq t_{i} \leq k-1,0<\chi_{i}<2 .
$$

If $M_{i}$ is a $2 r \times 2 r$ normal Jordan block belonging to $\lambda^{ \pm 1}$ of $\gamma(1)$ with $r \geq 2$, then the perturbation on $M_{i}$ defined in Step 2 produces $r$ times $2 \times 2$ rotational matrices $R\left(\theta_{j}\right)$ in the normal form of the $k$ th nondegenerate matrix $\gamma_{1}(1)$ as in (3.3) or (3.4) such that

$$
\theta_{j}=\frac{2 t_{j}+\chi_{j}}{k} \pi, \quad 1 \leq t_{j} \leq k-1,0<\chi_{j}<2,1 \leq j \leq r .
$$

From (8.25), where $r=1$, and (8.26), in both cases for the block $M_{i}$ we obtain

$$
\sum_{j=1}^{r} 2 t_{j} \geq \operatorname{dim} \operatorname{span}\left\{\text { eigenvectors belonging to } \lambda^{ \pm 1} \text { of } M_{i}\right. \text { \}. }
$$

Repeating this procedure for all eigenvalues of $\gamma(1)$ which are nonreal $k$ th roots of unity, we obtain that for the part $\gamma_{1}$ obtained in the first three steps, the part of $\mu^{+}$obtained from perturbations of these eigenvalues is not smaller than $\varphi_{k}$. Note that here $\sigma(\gamma(1))$ may contain other values on the unit circle which are not roots of unity.

$2^{\circ}$. If $k$ is even and $\omega_{0}>0$, then, as we discussed in (6.13), the eigenvectors $\xi_{j}, 1 \leq j \leq r$ for some $r \geq 1$, belonging to the eigenvalue -1 corresponding to the index $\omega_{0}$ can be perturbed to eigenvectors belonging to eigenvalues $\lambda_{j}^{ \pm 1}=$ $e^{ \pm \theta_{j} \sqrt{-1}} \in \mathbf{U} \backslash \mathbf{R}$ near -1 satisfying

$$
\theta_{j}=\frac{2 t_{j}+\chi_{j}}{k} \pi, \quad \text { with } t_{j} \geq\left[\frac{k}{2}\right] \geq 1 \text { and } 0<\chi_{j}<2 .
$$

$3^{\circ}$. If $k$ is even and $\omega_{+}>0$, similarly by (6.17), the eigenvectors $\xi_{j}, 1 \leq j \leq r$ for some $r \geq 1$, belonging to the eigenvalue -1 corresponding to the index $\omega_{+}$can be perturbed to eigenvectors belonging to eigenvalues $\lambda_{j}^{ \pm 1}=e^{ \pm \theta_{j} \sqrt{-1}} \in \mathbf{U} \backslash \mathbf{R}$ near -1 satisfying

$$
\theta_{j}=\frac{2 t_{j}+\chi_{j}}{k} \pi, \quad \text { with } t_{j} \geq 1 \text { and } 0<\chi_{j}<2 .
$$

Thus by $1^{\circ}-3^{\circ}$, we obtain that the path $\gamma_{1}$ obtained in such a way satisfies

$$
\sum_{j=1}^{\mu^{+}} 2 t_{j}^{+} \geq 2 \varphi_{k}+2 \omega_{0}+\omega_{+}=\nu_{k}-\nu_{1}-\frac{\left(1+(-1)^{k}\right)}{2} \omega .
$$

Here we have used Lemma 8.1. This proves $4^{\circ}$ for the perturbation path $\gamma_{1}$.

The proof of Theorem 8.3 is complete.

Corollary 8.4. Under the assumptions of Theorem 8.3, for any path $\beta$ sufficiently close to $\gamma$ and satisfying $\beta(T) \in \operatorname{Sp}(2 n)_{k}^{*}$,

$$
\begin{gathered}
i_{k T}(\tilde{\beta}) \geq i_{k T} \geq i_{k T}(\tilde{\beta})-\nu_{k T}, \\
i_{k T}(\tilde{\beta})=k\left(i_{T}(\beta)-\mu(\beta)\right)+\sum_{j=1}^{\mu(\beta)} 2 t_{j}(\beta)+\mu(\beta) .
\end{gathered}
$$

Proof. This follows from the proof for $1^{\circ}$ of Theorem 8.3. 


\section{Controlling the minimal period VIA MASLOV-TYPE INDICES}

For given $T>0$, in order to study the minimal period of a nonconstant $T$ periodic solution of a nonlinear autonomous Hamiltonian system (1.1) via its Maslov-type indices, we first consider the corresponding linearized system, and study the number of iterations of the definition interval of the system via its Maslovtype indices.

Let $B \in C\left(S_{T}, \mathcal{L}_{s}\left(\mathbf{R}^{2 n}\right)\right)$. Denote by $\gamma:[0, T] \rightarrow \operatorname{Sp}(2 n)$ the fundamental solution on the time interval $[0, T]$ of the linear system

$$
\dot{y}=J B(t) y \text {. }
$$

As we know, for given $k \in \mathbf{N}$, the path $\tilde{\gamma}:[0, k T] \rightarrow \operatorname{Sp}(2 n)$ defined by (4.2) is the fundamental solution of $(9.1)$ on the time interval $[0, k T]$. Denote by $\left(i_{T}, \nu_{T}\right)=$ $\left(i_{T}(\gamma), \nu_{T}(\gamma)\right)$ and $\left(i_{k T}, \nu_{k T}\right)=\left(i_{k T}(\tilde{\gamma}), \nu_{k}(\tilde{\gamma})\right)$ the corresponding Maslov-type indices of (9.1).

The first result of this section is the following theorem.

Theorem 9.1. Let $B \in C\left(S_{T}, \mathcal{L}_{s}\left(\mathbf{R}^{2 n}\right)\right)$. Suppose for some positive integer $k$ the following condition holds:

$$
n+1 \geq i_{k T}, \quad i_{T} \geq n, \quad \nu_{T} \geq 1 .
$$

Then $k=1$.

Proof. Without loss of generality, we assume $T=1$. We apply Theorem 8.3 to the system (9.1). Thus the path $\gamma$ can be perturbed to a nearby nondegenerate path $\beta$ such that the integers $\mu(\beta)$ and $t_{j}(\beta)$ with $1 \leq j \leq \mu(\beta)$, which are determined by a normal form of $\beta(1)$ in $\operatorname{Sp}(2 n)_{k}^{*}$ as we defined in $\S 3$, satisfy

$$
\begin{gathered}
\mu(\beta) \leq n-\omega, \\
\sum_{j=1}^{\mu(\beta)} 2 t_{j}(\beta) \geq \nu_{k}-\nu_{1}-\frac{1+(-1)^{k}}{2} \omega, \\
i_{k} \geq k\left(i_{1}+\nu_{1}-\mu(\beta)\right)+\sum_{j=1}^{\mu(\beta)} 2 t_{j}(\beta)+\mu(\beta)-\nu_{k},
\end{gathered}
$$

where $\omega=\omega(\gamma(1))$ is given by Definition 6.3 .

Note that (9.2) means that the perturbation path $\beta$ is obtained from $\gamma$ by perturbing all the normal Jordan blocks belonging to the eigenvalue -1 of $\gamma(1)$ corresponding to $\omega$ to nearby negative real eigenvalues of $\beta(1)$ away from -1 . (9.3) means that the perturbation path $\beta$ is obtained from $\gamma$ by perturbing all the normal Jordan blocks belonging to the eigenvalues of $\gamma(1)$ which are $k$ th roots of unity to eigenvalues of $\beta(1)$ suitably located on the unit circle away from the original values, and all the normal Jordan blocks belonging to the eigenvalue -1 of $\gamma(1)$ not corresponding to $\omega$ to nearby eigenvalues of $\beta(1)$ suitably located on the unit circle away from -1 . The existence of such a path $\beta$ is proved in Theorem 8.3.

We distinguish two cases.

Case 1. $k$ is odd. 
By (9.3) and the oddness of $k$, we obtain

$$
\sum_{j=1}^{\mu(\beta)} 2 t_{j}(\beta) \geq \nu_{k}-\nu_{1} .
$$

Combining this with (9.2), (9.4), and (M1) yields

$$
\begin{aligned}
n+1 \geq i_{k} & \geq k\left(i_{1}+\nu_{1}-\mu(\beta)\right)+\mu(\beta)-\nu_{1} \\
& \geq n+(k-1)\left(n+\nu_{1}-\mu(\beta)\right) \\
& \geq n+(k-1) \nu_{1} \\
& \geq n+(k-1) .
\end{aligned}
$$

Thus $k=1$.

Case 2. $k$ is even.

We carry out the proof of this case in two steps.

\section{Step 1.}

Claim. $k \leq 2$.

In fact, if $k \geq 4$, then by (9.3) and the evenness of $k$, we obtain

$$
\sum_{j=1}^{\mu(\beta)} 2 t_{j}(\beta) \geq \nu_{k}-\nu_{1}-\omega .
$$

Combining this with (9.2), (9.4), and (M1) yields

$$
\begin{aligned}
n+1 & \geq i_{k} \geq k\left(i_{1}+\nu_{1}-\mu(\beta)\right)+\mu(\beta)-\nu_{1}-\omega \\
& \geq 4\left(n+\nu_{1}-\mu(\beta)\right)+\mu(\beta)-\nu_{1}-\omega \\
& =\left(n+3 \nu_{1}\right)+(n-\mu(\beta))+(2 n-2 \mu(\beta)-\omega) \\
& \geq n+3 .
\end{aligned}
$$

This contradiction yields the claim.

\section{Step 2.}

Claim. $k \neq 2$.

We argue indirectly, and assume $k=2$. By (9.2)-(9.4) and (M1), we obtain

$$
\begin{aligned}
n+1 \geq i_{2} & \geq 2\left(i_{1}+\nu_{1}-\mu(\beta)\right)+\sum_{j=1}^{\mu(\beta)} 2 t_{j}(\beta)+\mu(\beta)-\nu_{2} \\
& \geq 2\left(i_{1}+\nu_{1}-\mu(\beta)\right)+\mu(\beta)-\nu_{1}-\omega \\
& \geq n+\nu_{1}+(n-\mu(\beta)-\omega) \\
& \geq n+1 .
\end{aligned}
$$

Thus, by (9.2), we must have

$$
\begin{gathered}
i_{1}=n, \quad \nu_{1}=1, \\
i_{2}=i_{2}(\tilde{\beta})-\nu_{2}=i_{1}(\beta)=n+1, \quad \nu_{1}(\beta)=0, \\
\mu(\beta)+\omega=n .
\end{gathered}
$$


Note that $\omega=\omega_{2}+\omega_{-}$. By the definition of the path $\beta$, the eigenvalue -1 corresponding to $\omega_{2}$ is perturbed to nearby real negative eigenvalues on $\mathbf{R} \backslash\{-1\}$. Thus (9.8) and the definition of $\omega_{2}$ imply that every normal form $M_{i} \in \operatorname{Sp}\left(2 k_{i}\right)$ in (6.22) corresponding to $\omega_{2}$ must satisfy $k_{i}=2$ and $\operatorname{dim} \operatorname{ker}\left(M_{i}+I\right)=2$.

Now we consider a second nondegenerate perturbation path $\zeta$ of $\gamma$ on $[0,1]$ that $\zeta$ is obtained in the same way as $\beta$ except that this time we perturb all the normal form blocks belonging to the eigenvalue -1 of $\gamma(1)$ corresponding to $\omega$ to nearby eigenvalues on $\mathbf{U} \backslash \mathbf{R}$ of $\zeta(1)$ away from -1 so that $\zeta(1)$ has only normal forms of $\diamond$-product of $R(\theta)$ 's. This can be realized for any normal form $M_{i}$ corresponding to $\omega_{2}$ via perturbations in (6.7) and (7.11), and for any normal form $M$ corresponding to $\omega_{-}$via perturbations in the subcase 4.3 of $\S 6$. We keep such perturbations for the path $\zeta$.

Thus by (9.8) for this path $\zeta$ we have

$$
\mu(\zeta)=\mu(\beta)+\omega=n
$$

By (9.7),

$$
i_{1}(\zeta)=i_{1}(\beta)=n+1, \quad \nu_{1}(\zeta)=0 .
$$

Since $\mu(\zeta)=n$ and $i_{1}(\zeta)=n+1$ have different parities, this violates Theorem 4.1. Therefore the claim holds.

These two steps of Case 2 show that $k$ cannot be even under the condition (M1). Thus we must have $k=1$, and the proof is complete.

Corollary 9.2. Let $B \in C\left(S_{T}, \mathcal{L}_{s}\left(\mathbf{R}^{2 n}\right)\right)$. Suppose for some positive integer $k$ the following condition holds:

$$
i_{T}+1 \geq i_{k T}, \quad i_{T} \geq n, \quad \nu_{T} \geq 1 .
$$

Then $k=1$.

Proof. Set $T=1$. As in (9.4) we obtain

$$
\begin{aligned}
& \left(i_{1}-n\right)+n+1 \geq i_{k} \\
& \quad \geq k\left(i_{1}-n\right)+k\left(n+\nu_{1}-\mu(\beta)\right)+\sum_{j=1}^{\mu(\beta)} 2 t_{j}(\beta)+\mu(\beta)-\nu_{k} .
\end{aligned}
$$

Then, similarly to the proof of Theorem 9.1 , we get $k=1$.

A direct consequence of Theorem 9.1 is the following theorem on controlling the minimal period of a given $T$-periodic solution $x_{0}$ of the nonlinear autonomous Hamiltonian system (1.1) via the estimates of Maslov-type indices of $x_{0}$.

Theorem 9.3. Suppose the following condition holds:

(H1) $H \in C^{2}\left(\mathbf{R}^{2 n}, \mathbf{R}\right)$.

For $T>0$, let $x_{0} \in C^{2}\left(S_{T}, \mathbf{R}^{2 n}\right)$ be a $T$-periodic solution of the system (1.1) with minimal period $T / k$ for some $k \in \mathbf{N}$. Let the Maslov-type indices of $x_{0}$ satisfy the following conditions:

(X1) $i_{T}\left(x_{0}\right) \leq n+1$.

(X2) $i_{T / k}\left(x_{0}\right) \geq n$.

Then $k=1$, i.e. the solution $x_{0}$ possesses minimal period $T$. 
Proof. Let $\tau=T / k$. Then (X1) and (X2) imply $i_{k \tau}\left(x_{0}\right) \leq n+1$ and $i_{\tau}\left(x_{0}\right) \geq$ $n$. Since $x_{0}$ is a nonconstant $\tau$-periodic solution of (1.1), the function $\dot{x}_{0}$ is a nonconstant $\tau$-periodic solution of the linear system

$$
\dot{y}=J H^{\prime \prime}\left(x_{0}(t)\right) y .
$$

Thus $\nu_{\tau}\left(x_{0}\right) \geq 1$. Now we can apply Theorem 9.1 to conclude that $k=1$.

Corollary 9.4. Suppose the condition (H1) holds. For $T>0$, let $x_{0} \in C^{2}\left(S_{T}, \mathbf{R}^{2 n}\right)$ be a nonconstant T-periodic solution of (1.1) which satisfies (X1) and the following conditions:

(HX1) $H^{\prime \prime}\left(x_{0}(t)\right) \geq 0$ for every $t \in \mathbf{R}$.

(HX2) $\int_{0}^{T} H^{\prime \prime}\left(x_{0}(t)\right) d t$ is positive definite.

Then $k=1$, i.e. the solution $x_{0}$ possesses minimal period $T$.

Proof. Suppose that $x_{0}$ has minimal period $T / k$ for some $k \in \mathbf{N}$. Let $\tau=T / k$.

In the saddle point reduction described in $\S \S 2$ and 8 for the Hamiltonian function $K(t, y)=\frac{1}{2} H^{\prime \prime}\left(x_{0}(t)\right) y \cdot y$, the space $L=L^{2}\left(S_{\tau}, \mathbf{R}^{2 n}\right)$ possesses an orthogonal decomposition

$$
L=L^{+} \oplus L^{0} \oplus L^{-}, \quad L^{0}=\mathbf{R}^{2 n},
$$

such that $\langle A x, x\rangle$ is positive, null, or negative definite on $L^{+}, L^{0}$, or $L^{-}$respectively, where $A=-J \frac{d}{d t}$. Let $P \pm: L \rightarrow L^{ \pm}$be the projectors. Correspondingly, the finitedimensional space $Z$ has an orthogonal decomposition

$$
Z=Z^{+} \oplus Z^{0} \oplus Z^{-}, \quad Z^{0}=\mathbf{R}^{2 n} .
$$

Denote the functional corresponding to $K$ by $a: Z \rightarrow \mathbf{R}$. In [AZ], the following inequality is proved (cf. (7.3) of [AZ]):

$$
\begin{aligned}
a(z) \leq & \frac{1}{2}\left\langle A\left(P_{-} v(z)+z\right), P_{-} v(z)+z\right\rangle \\
& -\frac{1}{2} \int_{0}^{\tau} H^{\prime \prime}\left(x_{0}(t)\right)\left(P_{-} v(z)+z\right) \cdot\left(P_{-} v(z)+z\right) d t \quad \forall z \in Z .
\end{aligned}
$$

By (9.14), we obtain that for any $z=z_{-}+z_{0} \in Z^{-} \oplus Z^{0} \backslash\{0\}$

$$
\begin{aligned}
a(z) \leq & -\frac{1}{2}\left\|P_{-} v(z)+z_{-}\right\|^{2} \\
& -\frac{1}{2} \int_{0}^{\tau} H^{\prime \prime}\left(x_{0}(t)\right)\left(P_{-} v(z)+z_{-}+z_{0}\right) \cdot\left(P_{-} v(z)+z_{-}+z_{0}\right) d t \\
= & -\frac{1}{2}\left(\left\|P_{-} v(z)\right\|^{2}+\left\|z_{-}\right\|^{2}\right) \\
& -\frac{1}{2} \int_{0}^{\tau} H^{\prime \prime}\left(x_{0}(t)\right)\left(P_{-} v(z)+z_{-}+z_{0}\right) \cdot\left(P_{-} v(z)+z_{-}+z_{0}\right) d t .
\end{aligned}
$$

Thus if $\left\|P_{-} v(z)\right\|^{2}+\left\|z_{-}\right\|^{2}>0$, by (HX1) we have $a(z)<0$. On the other hand, if $\left\|P_{-} v(z)\right\|^{2}+\left\|z_{-}\right\|^{2}=0,(9.15)$ becomes

$$
\begin{aligned}
a(z) & \leq-\frac{1}{2}\left(\int_{0}^{\tau} H^{\prime \prime}\left(x_{0}(t)\right) d t\right) z_{0} \cdot z_{0} \\
& =-\frac{1}{2 k}\left(\int_{0}^{T} H^{\prime \prime}\left(x_{0}(t)\right) d t\right) z_{0} \cdot z_{0}<0 .
\end{aligned}
$$


Here in the last step we have used (HX2). Thus the negative Morse index $m^{-}$of the functional $a$ satisfies

$$
m^{-} \geq \operatorname{dim} Z_{-}+\operatorname{dim} Z_{0}=(d-n)+2 n=d+n .
$$

Combining this with Theorem 2.7, we obtain the condition (X2):

$$
i_{\tau}\left(x_{0}\right) \geq n \text {. }
$$

Now we can apply Theorem 9.3 to conclude that $k=1$, and complete the proof.

\section{Applications to autonomous nonlinear Hamiltonian systems}

In this section we apply our results to autonomous asymptotically linear Hamiltonian systems defined on $\mathbf{R}^{2 n}$,

$$
\dot{x}=J H^{\prime}(x) .
$$

For $T>0$, define

$$
\mathcal{S}_{T}(H)=\left\{x \in C^{1}\left(S_{T}, \mathbf{R}^{2 n}\right) \mid x \not \equiv \text { constant, } x \text { is a solution of }(10.1)\right\} .
$$

The main result in this section is the following theorem.

Theorem 10.1. Suppose the Hamiltonian function $H$ satisfies the following conditions:

(H1) $H \in C^{2}\left(\mathbf{R}^{2 n}, \mathbf{R}\right)$.

(H2) There exists a positive definite matrix $B \in \mathcal{L}_{s}\left(\mathbf{R}^{2 n}\right)$ such that

$$
H^{\prime}(x)=B x+o(|x|) \quad \text { as }|x| \rightarrow \infty .
$$

(H3) $H(x)=o\left(|x|^{2}\right)$ near $x=0$.

(H4) $H(x) \geq 0$ for all $x \in \mathbf{R}^{2 n}$.

Suppose $T>0$ and the following conditions hold:

(HT1) $H^{\prime \prime}(x(t)) \geq 0$ for every $x \in \mathcal{S}_{T}(H)$ and $t \in \mathbf{R}$.

(HT2) $\int_{0}^{T} H^{\prime \prime}(x(t)) d t$ is positive definite for every $x \in \mathcal{S}_{T}(H)$.

(HT3) $\nu_{T}(B)=0$.

(HT4) $i_{T}(B)>n$.

Then the system (10.1) possesses a solution $x$ with minimal period $T$.

In order to prove Theorem 10.1, we need the following well-known Palais-Smale condition and the saddle point theorem.

Definition 10.2. A $C^{1}$ real functional $f$ defined on a real Hilbert space $E$ is said to satisfy the Palais-Smale condition (PS) on $E$, if for every sequence $\left\{x_{k}\right\} \subset E$ the conditions $\left\{\left|f\left(x_{k}\right)\right|\right\}$ bounded and $f^{\prime}\left(x_{k}\right) \rightarrow 0$ as $k \rightarrow \infty$ imply that $\left\{x_{k}\right\}$ possesses a convergent subsequence.

Theorem 10.3. Let $E$ be a real Hilbert space with orthogonal decomposition $E=$ $X \oplus Y$, where $\operatorname{dim} X<\infty$. Suppose $f \in C^{2}(E, \mathbf{R})$, satisfies (PS) and the following conditions.

(F1) There exist $\rho$ and $\alpha>0$ such that $f(w) \geq \alpha \forall w \in \partial B_{\rho}(0) \cap Y$.

(F2) There exist $e \in \partial B_{1}(0) \cap Y$ and $R>\rho$ such that $f(w) \leq 0 \forall w \in \partial Q$, where $Q=\left(\overline{B_{R}(0)} \cap X\right) \oplus\{r e \mid 0 \leq r \leq R\}$.

Then: 
$1^{\circ}$. $f$ possesses a critical value $c \geq \alpha$, which is given by

$$
c=\inf _{h \in \Gamma} \max _{w \in Q} f(h(w)),
$$

where $\Gamma=\{h \in C(\bar{Q}, E) \mid h=$ id on $\partial Q\}$.

$2^{\circ}$. There exists an element $w_{0} \in \mathcal{K}_{c} \equiv\left\{w \in E \mid f^{\prime}(w)=0, f(w)=c\right\}$ such that the negative Morse index $m^{-}\left(w_{0}\right)$ of $f$ at $w_{0}$ satisfies

$$
m^{-}\left(w_{0}\right) \leq \operatorname{dim} X+1 .
$$

Remark 10.4. The proof of this theorem can be found in [Ra2], [Gh], [LS], and [So].

Proof of Theorem 10.1. We carry out the proof in several steps.

Step 1. In order to use the saddle point reduction method, we need to truncate the function $H$ suitably to get the boundedness of $\left\|H^{\prime \prime}\right\|_{C}$.

We note that conditions (H2) and (HT4) imply that

$$
H(x)=\frac{1}{2} B x \cdot x+o\left(|x|^{2}\right) \quad \text { as }|x| \rightarrow \infty,
$$

and the existence of constants $\Lambda_{0} \geq \lambda_{0}>0$ such that

$$
\Lambda_{0} I \geq B \geq \lambda_{0} I .
$$

Thus there exists a constant $k_{0} \geq 5$ such that

$$
2 \Lambda_{0}|x|^{2} \geq H(x) \geq \frac{\lambda_{0}}{4}|x|^{2} \quad \forall|x| \geq k_{0} .
$$

Claim. For any integer $k \geq k_{0}$, there exist a constant $b(k) \geq 1$ and a function $\chi_{k} \in C^{2}([0,+\infty),[0,1])$ such that

$$
\begin{gathered}
\chi_{k}(r)=1 \quad \text { for } 0 \leq r \leq k, \\
\chi_{k}(r)=0 \quad \text { for } k+b(k) \leq r, \\
0 \leq-\chi_{k}^{\prime}(r) \leq 2 / r \quad \text { for } k<r<k+b(k) .
\end{gathered}
$$

In fact, we first define $\chi_{k}=1$ for $r \in[0, k]$. Then, by induction on integers $m \geq 0$, we can assume that $\chi_{k}$ has been extended smoothly to $[0, k+m]$ so that

$$
\begin{aligned}
& 0 \leq \chi_{k}(k+m) \leq \max \left\{0,1-\sum_{i=1}^{m} \frac{1}{k+i}\right\} \equiv M(k, m), \\
& 0 \leq-\chi_{k}^{\prime}(k+m)<\frac{2}{k+m+1}, \\
& 0 \leq-\chi_{k}^{\prime}(r)<\frac{1}{k+m} \leq \frac{2}{r} \text { for } k+m-1<r \leq k+m .
\end{aligned}
$$

Since $\sum_{i>1} \frac{1}{k+i}=+\infty$, there exists an integer $b(k) \geq 3$ such that $M(k, b(k)-1)=0$ and $M(k, b(k)-2)>0$. Then we slightly modify $\chi_{k}$ near $k+b(k)-1$ and extend it to $(k+b(k)-1, k+b(k)]$ so that $(10.10)$ holds for $m=k+b(k)$ and $\chi_{k}(k+b(k))=$ $\chi_{k}^{\prime}(k+b(k))=\chi_{k}^{\prime \prime}(k+b(k))=0$. Finally we extend $\chi_{k}$ to $(k+b(k),+\infty)$ by $(10.8)$, and obtain the claim.

For $k \geq k_{0}$ define

$$
H_{k}(x)=\chi_{k}(|x|) H(x)+\frac{1}{2}\left(1-\chi_{k}(|x|)\right) B x \cdot x \quad \forall x \in \mathbf{R}^{2 n} .
$$


Then by (10.5), (10.6), and (10.9), for any $k \geq k_{0}$

$$
\begin{gathered}
2 \Lambda_{0}|x|^{2} \geq H_{k}(x) \geq \frac{\lambda_{0}}{4}|x|^{2} \quad \forall|x| \geq k_{0}, \\
2 \Lambda_{0}|x|^{2}+K \geq H_{k}(x) \geq \frac{\lambda_{0}}{4}|x|^{2}-K \quad \forall x \in \mathbf{R}^{2 n},
\end{gathered}
$$

and

$$
\begin{aligned}
\left|H_{k}^{\prime}(x)-B x\right| & \leq\left|\chi_{k}(|x|)\left(H^{\prime}(x)-B x\right)\right|+\left|\chi_{k}^{\prime}(|x|)\left(H(x)-\frac{1}{2} B x \cdot x\right) \frac{x}{|x|}\right| \\
& \leq\left|H^{\prime}(x)-B x\right|+\frac{2}{|x|}\left|H(x)-\frac{1}{2} B x \cdot x\right| \quad \forall|x|>0
\end{aligned}
$$

where $K=\max _{|x| \leq k_{0}} H(x)$. Thus by (H2) for $H$ and (10.4) the function $H_{k}$ satisfies (H2) for the same $B \in \mathcal{L}_{s}\left(\mathbf{R}^{2 n}\right)$, and by (10.14), $H_{k}^{\prime}(x)$ converges to $B x$ as $|x| \rightarrow \infty$ uniformly for all $k \geq k_{0}$. Note that for each $k \geq k_{0}$, the function $H_{k}$ satisfies (H1)-(H4), (HT3), and (HT4), and

$$
\left\|H_{k}^{\prime \prime}(x)\right\|_{C\left(\mathbf{R}^{2 n}\right)}<+\infty .
$$

In Steps 2-6, we shall fix $k \geq k_{0}$, and prove the existence of a special $T$-periodic solution of the Hamiltonian system

$$
\dot{x}=J H_{k}^{\prime}(x) .
$$

For notational simplicity, in these steps we shall omit the subscript $k$.

Step 2. By (H1) and (10.15), using the saddle point reduction method described in $\S 2$ (cf. [AZ]), for the Hilbert space $L=L^{2}\left(S_{T}, \mathbf{R}^{2 n}\right)$, we obtain the functional

$$
f(x)=\frac{1}{2}\langle A x, x\rangle_{L^{2}}-\int_{0}^{T} H(x) d t
$$

defined on $E=\operatorname{dom} A=W^{1,2}\left(S_{T}, \mathbf{R}^{2 n}\right) \subset L$, and the $C^{2}$ functional

$$
\begin{aligned}
a(z)=f(u(z)) & =\frac{1}{2}\langle A u(z), u(z)\rangle_{L^{2}}-\int_{0}^{T} H(u(z)) d t \\
& =\frac{1}{2}\left\|P_{+} u(z)\right\|^{2}-\frac{1}{2}\left\|P_{-} u(z)\right\|^{2}-\int_{0}^{T} H(u(z)) d t
\end{aligned}
$$

defined on the finite-dimensional space $Z$ with $2 d=\operatorname{dim} Z$. Here $A=-J d / d t$, and $\|\cdot\|$ is the $W^{1 / 2,2}\left(S_{T}, \mathbf{R}^{2 n}\right)$ norm, and $u \in C^{1}(Z, E)$ is the injective map given by the reduction method. Depending on whether the quadratic form $\langle A x, x\rangle_{L^{2}}$ is positive, null, or negative definite, we obtain orthogonal decompositions

$$
L=L^{+} \oplus L^{0} \oplus L^{-}, \quad Z=Z^{+} \oplus Z^{0} \oplus Z^{-} .
$$

Denote by $P_{ \pm}: L \rightarrow L^{ \pm}, P_{0}: L \rightarrow L^{0}$, and $P: L \rightarrow Z$ the projectors. Then $u(z)=v(z)+z$ with $P v(z)=0$. In order to apply Theorem 10.3 to the functional $a$ on $Z$, let $X=Z^{0} \oplus Z^{-}$and $Y=Z^{+}$.

Step 3. By conditions (H2) and (HT3), it is well known that the functionals $f$ and $a$ satisfy the Palais-Smale condition on $E$ and $Z$ respectively. For details we refer to $[\mathrm{CZ}],[\mathrm{LZ}]$, and [Lo7]. 
Step 4. By the condition (H3), we can apply (7.2) of [AZ] to obtain

$$
a(z) \geq \frac{1}{2}\left\langle A\left(P_{+} v(z)+z\right), P_{+} v(z)+z\right\rangle_{L^{2}}+o\left(\|z\|_{L^{2}}^{2}\right) \quad \text { as } z \rightarrow 0 \text { in } Z .
$$

Thus there exists $\rho>0$ small enough so that

$$
\begin{aligned}
a(z) & \geq \frac{1}{2}\left\|P_{+} v(z)+z\right\|^{2}+o\left(\|z\|_{L^{2}}^{2}\right) \\
& =\frac{1}{2}\left(\left\|P_{+} v(z)\right\|^{2}+\|z\|^{2}\right)+o\left(\|z\|_{L^{2}}^{2}\right) \\
& \geq \frac{1}{4}\|z\|^{2}=\frac{1}{4} \rho^{2}>0 \quad \forall\|z\|=\rho, z \in Z^{+} .
\end{aligned}
$$

Thus condition (F1) holds.

Step 5. By condition (HT4), there exist an element $y \in Z^{+}$with $\|y\|=1$ and $\lambda_{1}>0$ such that

$$
(A-B) y=-\lambda_{1} y \text { in } E,
$$

where $\langle B x, x\rangle_{L^{2}}=\int_{0}^{T} B x(t) \cdot x(t) d t$ for $x \in E$. For $R>0$ large (to be determined later) we define

$$
\begin{array}{r}
Q=\left\{z=r y+z_{0}+z_{-} \in Z \mid z_{0}+z_{-} \in Z^{0} \oplus Z^{-},\right. \\
\left.\left\|z_{0}+z_{-}\right\| \leq R, 0 \leq r \leq R\right\} .
\end{array}
$$

Then by (7.3) of $[\mathrm{AZ}]$ we obtain

$$
\begin{aligned}
a(z) \leq & \frac{1}{2}\left\langle A\left(P_{-} v(z)+z\right), P_{-} v(z)+z\right\rangle_{L^{2}} \\
& -\int_{0}^{T} H\left(P_{-} v(z)+z\right) d t \quad \forall z \in Z .
\end{aligned}
$$

Thus

$$
\begin{aligned}
a(z) & \leq-\frac{1}{2}\left\|P_{-} v(z)+z_{-}\right\|^{2}-\int_{0}^{T} H\left(P_{-} v(z)+z\right) d t \\
& \leq 0 \quad \forall z=z_{0}+z_{-} \in Z^{0} \oplus Z^{-} .
\end{aligned}
$$

For $z=r y+z_{0}+z_{-} \in \partial Q$ we have

$$
\begin{aligned}
a(z) \leq & \frac{1}{2}\left\langle A\left(P_{-} v(z)+z\right), P_{-} v(z)+z\right\rangle_{L^{2}}-\int_{0}^{T} H\left(P_{-} v(z)+z\right) d t \\
= & \frac{1}{2}\left\langle A\left(P_{-} v(z)+r y+z_{-}\right), P_{-} v(z)+r y+z_{-}\right\rangle_{L^{2}} \\
& -\frac{1}{2} \int_{0}^{T} B\left(P_{-} v(z)+z\right) \cdot\left(P_{-} v(z)+z\right) d t+o\left(\left\|P_{-} v(z)+z\right\|_{L^{2}}^{2}\right) .
\end{aligned}
$$

Here we have used (H2). Thus

$$
\begin{aligned}
a(z) \leq & -\frac{1}{2}\left\|P_{-} v(z)+z_{-}\right\|^{2}+\frac{r^{2}}{2}\langle(A-B) y \cdot y\rangle_{L^{2}} \\
& -r \int_{0}^{T} B y \cdot\left(P_{-} v(z)+z_{0}+z_{-}\right) d t \\
& -\frac{1}{2} \int_{0}^{T} B\left(P_{-} v(z)+z_{0}+z_{-}\right) \cdot\left(P_{-} v(z)+z_{0}+z_{-}\right) d t \\
& +o\left(\left\|P_{-} v(z)+z\right\|_{L^{2}}^{2}\right) .
\end{aligned}
$$


Note that $x=P_{-} v(z)+z_{0}+z_{-}$is orthogonal to $y$ and $A y$ in $L^{2}$. We obtain

$$
\begin{aligned}
\int_{0}^{T} B y \cdot x d t & =-\int_{0}^{T}(A-B) y \cdot x d t+\int_{0}^{T} A y \cdot x d t \\
& =\lambda_{1} \int_{0}^{T} y \cdot x d t=0 .
\end{aligned}
$$

By (10.5), (10.22), (10.26) and (10.27) we have

$$
\begin{aligned}
a(z) \leq & -\frac{1}{2}\left\|P_{-} v(z)+z_{-}\right\|^{2}-\lambda_{1} \frac{r^{2}}{2}\|y\|_{L^{2}}^{2} \\
& -\frac{\lambda_{0}}{2}\left\|P_{-} v(z)+z_{0}+z_{-}\right\|^{2}+o\left(\left\|P_{-} v(z)+z\right\|_{L^{2}}^{2}\right) \\
\leq & -\frac{1}{2} \min \left\{1, \lambda_{0}, \lambda_{1}\right\}\left\|P_{-} v(z)+z\right\|_{L^{2}}^{2}+o\left(\left\|P_{-} v(z)+z\right\|_{L^{2}}^{2}\right) .
\end{aligned}
$$

Thus by taking $R>0$ to be large enough we obtain

$$
a(z) \leq 0 \quad \forall z=r y+z_{0}+z_{-} \in \partial Q \text { with }\left\|z_{0}+z_{-}\right\|=R \text { or } r=R .
$$

Combining (10.25) and (10.29) yields the condition (F2).

Step 6. Now we can apply Theorem 10.3 to obtain a critical point $z \in Z$ of $a$ with $a(z) \geq \frac{1}{4} \rho^{2}>0$, and the Morse index $m^{-}(x)$ of $a$ with $x=u(z)$ satisfying

$$
m^{-}(x) \leq \operatorname{dim}\left(Z^{0} \oplus Z^{-}\right)+1=(d-n)+2 n+1=d+n+1 .
$$

Note that $a(z)>0$ implies $z \not \equiv$ constant, and then $x \not \equiv$ constant. By (H1), (10.14) and Theorem 2.7 we obtain

$$
i_{T}(x) \leq n+1 .
$$

Thus condition (X1) holds.

So for each $k \geq k_{0}$, the above proof yields a nonconstant $T$-periodic solution $x_{k}$ of (10.16) which satisfies (10.31).

\section{Step 7.}

Claim. There exists a constant $k_{1} \geq k_{0}$ such that for any $k \geq k_{1}$, if $x_{k}$ is a $T$-periodic solution of the system (10.16), then it is also a T-periodic solution of (10.1).

In fact, by (HT3) there exists a constant $\alpha>0$ such that

$$
\|(A-B) y\|_{L^{2}} \geq 2 \alpha\|y\|_{L^{2}} \quad \forall y \in E .
$$

Fix $k \geq k_{0}$. Let $g_{k}(y)=\int_{0}^{T} H_{k}(y(t)) d t$ for $y \in L$. Then $g_{k}$ is $C^{1}$ on $L$. By (H2) and (10.13), as proved in $\S \S 5$ and 12 of [AZ], we obtain

$$
\left\|g_{k}^{\prime}(y)-B y\right\|_{L^{2}} /\|y\|_{L^{2}} \rightarrow 0
$$

for $\|y\|_{L^{2}} \rightarrow \infty$, and $y \in L$ uniformly in $k \geq k_{0}$. Thus there exists a constant $k_{2} \geq k_{0}$, independent of the choice of $k$, such that

$$
\left\|g_{k}^{\prime}(y)-B y\right\|_{L^{2}} \leq \alpha\|y\|_{L^{2}} \quad \forall\|y\|_{L^{2}} \geq k_{2}, y \in L .
$$

Denote by $f_{k}$ the functional defined by (10.17) with respect to $H_{k}$. Combining (10.32) and (10.34), we obtain

$$
\begin{aligned}
\left\|f_{k}^{\prime}(y)\right\|_{L^{2}} & \geq\|(A-B) y\|_{L^{2}}-\left\|g_{k}^{\prime}(y)-B y\right\|_{L^{2}} \\
& \geq \alpha\|y\|_{L^{2}} \quad \forall\|y\|_{L^{2}} \geq k_{2}, y \in E .
\end{aligned}
$$


Thus for any $k \geq k_{0}$ and any critical point $x_{k} \in E$ of $f_{k}$ we must have

$$
\left\|x_{k}\right\|_{L^{2}} \leq k_{2} \text {. }
$$

That is, for any $k \geq k_{0}$, every $T$-periodic solution $x_{k}$ of (10.16) must satisfy (10.36). Then by (10.13), (10.16), and (10.36), for every $t \in \mathbf{R}$

$$
\begin{aligned}
T H_{k}\left(x_{k}(t)\right) & =\int_{0}^{T} H_{k}\left(x_{k}(s)\right) d s \\
& \leq T K+2 \Lambda_{0} \int_{0}^{T}\left|x_{k}(s)\right|^{2} d s \\
& \leq T K+2 \Lambda_{0} k_{2}^{2} .
\end{aligned}
$$

Combining (10.37) with (10.13) again yields

$$
T \frac{\lambda_{0}}{5}\left|x_{k}(t)\right|^{2} \leq K+T K+2 \Lambda_{0} k_{2}^{2} \quad \forall t \in \mathbf{R} .
$$

This yields a uniform estimate of the $C$-norm for all $T$-periodic solutions of (10.16) with $k \geq k_{0}$ :

$$
\left\|x_{k}(\cdot)\right\|_{C\left(S_{T}\right)} \leq 1+\sqrt{\frac{5}{T \lambda_{0}}\left(K+T K+2 \Lambda_{0} k_{2}^{2}\right)} .
$$

Setting $k_{1}$ equal to the right-hand side of (10.39) proves the claim.

Step 8. The above proof yields a nonconstant $T$-periodic solution $x$ of (10.1), which satisfies (10.31), i.e. (X1). Note that (HT1) and (HT2) imply (HX1) and (HX2). Thus by Corollary 9.4 the solution $x$ possesses minimal period $T$.

The proof is complete.

The following corollary gives more accessible sufficient conditions for the existence of solutions with prescribed minimal period.

Corollary 10.5. For $T>0$, suppose the Hamiltonian function $H$ satisfies (H1)(H3), (HT3), (HT4), and the following conditions:

(H5) $H^{\prime \prime}(x) \geq 0$ for all $x \in \mathbf{R}^{2 n}$.

(H6) The set $D=\left\{x \in \mathbf{R}^{2 n} \mid H^{\prime}(x) \neq 0,0 \in \sigma\left(H^{\prime \prime}(x)\right)\right\}$ is hereditarily disconnected., i.e. every connected component of $D$ contains only one point.

Then the system (10.1) possesses a T-periodic solution $x$ with $T$ as its minimal period.

Proof. Note that (H4) follows from (H1), (H3), and (H5). (HT1) follows from (H5). Let $x \in \mathcal{S}_{T}(H)$. Since $x \not \equiv$ constant, $H^{\prime}(x(t)) \neq 0$ for all $t \in \mathbf{R}$. Thus $\left\{x(t) \mid 0 \in \sigma\left(H^{\prime \prime}(x(t))\right), t \in \mathbf{R}\right\}$ is a subset of $D$, hence is hereditarily disconnected. This implies (HT2). Now we can apply Theorem 10.1 to complete the proof.

Remark 10.6. In Theorem IV.4 of [EH], the same conclusion of Corollary 10.5 is proved under (H1)-(H3), (HT3), (HT4), and the condition

(H7) $H^{\prime \prime}(x) \geq g(x) I$ for all $x \neq 0$, where $g$ is some continuous function on $\mathbf{R}^{2 n}$ with $g(x)>0$ for $x \neq 0$.

Clearly our conditions (H5) and (H6) are weaker than (H7). For example, (H5) and (H6) allow the Hamiltonian function $H$ to be identically zero near the origin, and allow $H^{\prime \prime}(x)=0$ on some points in $\mathbf{R}^{2 n}$. Thus our Corollary 10.5 gives a strict generalization of Theorem IV.4 of $[\mathrm{EH}]$. 


\section{Applications to Hamiltonian systems on $\mathbf{R}^{2}$}

In this section we apply the iteration formula for the Maslov-type index theory to Hamiltonian systems defined on $\mathbf{R}^{2}$ with no convexity type conditions, and exhibit some counterexamples in higher-dimensional cases. We consider first the linear Hamiltonian system

$$
\dot{x}=J B(t) x, \quad x \in \mathbf{R}^{2} .
$$

For $T>0$, denote by $\gamma:[0, T] \rightarrow \operatorname{Sp}(2)$ the fundamental solution, and by $\left(i_{T}, \nu_{T}\right)=$ $\left(i_{T}(\gamma), \nu_{T}(\gamma)\right)$ the Maslov-type index of (11.1). One of the main results in this section is the following theorem.

Theorem 11.1. For $T>0$ and $k \in \mathbf{N}$, suppose $B \in C\left(S_{T}, \mathcal{L}_{s}\left(\mathbf{R}^{2}\right)\right)$, and

$$
2-\nu_{k T} \leq i_{k T} \leq 2 \text { and } \nu_{T} \geq 1 \text {. }
$$

Then $k=1$.

Proof. Without loss of generality, we suppose $T=1$. Since $\nu_{1} \geq 1$, as in $\S 2$, we define two nondegenerate perturbation paths $\gamma^{ \pm} \equiv \gamma_{ \pm 1}$ of $\gamma$ by (2.4). Using the notation defined in the earlier sections, we set $\mu^{ \pm} \equiv \mu\left(\gamma^{ \pm}\right)$, and if this number is positive, the matrix $\gamma^{ \pm}(1)$ has a normal form $R\left(\theta^{ \pm}\right)$, where

$$
\theta^{ \pm}=\frac{2 t^{ \pm}+\chi^{ \pm}}{k} \pi, \quad 0 \leq t^{ \pm} \leq k-1,0<\chi^{ \pm}<2 .
$$

Set $i_{1}^{ \pm} \equiv i_{1}\left(\gamma^{ \pm}\right)$, and denote by $\tilde{\gamma}^{ \pm}$the extension of $\gamma^{ \pm}$to the interval $[0, k]$.

By the discussion in [Lo2], $\operatorname{Sp}(2)^{0} \backslash\{I\}$ consists of two path-connected components each of which is homeomorphic to $\mathbf{R}^{2} \backslash\{0\}$. Denote them by

$$
\operatorname{Sp}(2)_{ \pm}^{0}=\left\{M \in \operatorname{Sp}(2)^{0} \backslash\{I\} \mid \sigma(M R(\theta)) \subset \mathbf{U} \backslash \mathbf{R} \text { for } 0< \pm \theta<\frac{\pi}{2}\right\} .
$$

To continue the proof we distinguish three cases:

Case 1. $\gamma(1)=I$. In this case, we have $\nu_{1}=\nu_{k}=2$. By direct computations (cf. [Lo1, Lo2]), we obtain

$$
\begin{aligned}
& \mu^{+}=1, \quad t^{+}=0, \quad i_{1}^{+}=i_{1}+\nu_{1}=i_{1}+2, \\
& \mu^{-}=1, \quad t^{-}=k-1, \quad i_{1}^{-}=i_{1} .
\end{aligned}
$$

Note that the values of $t^{ \pm}$can also be obtained directly from the discussion in [Lo2]. Thus by $1^{\circ}$ of Theorem 8.3 we have

$$
k\left(i_{1}^{-}-\mu^{-}\right)+2 t^{-}+\mu^{-} \geq i_{k} \geq k\left(i_{1}^{+}-\mu^{+}\right)+2 t^{+}+\mu^{+}-\nu_{k} .
$$

Together with (11.2) and (11.3) we obtain

$$
i_{k}=k\left(i_{1}+1\right)-1 \text {. }
$$

By the conditions (M3) and $\nu_{k}=2$ we obtain

$$
0 \leq i_{k}=k\left(i_{1}+1\right)-1 \leq 2 .
$$

This implies that

$$
1 \leq k\left(i_{1}+1\right) \leq 3 .
$$

Thus we must have $i_{1} \geq 0$ and $k \leq 3$. If $i_{1}=0$, then (11.1) yields $\mu^{+}=1$ and $i_{1}^{+}=2$. This contradicts Theorem 4.1. Therefore $i_{1} \geq 1$. By (11.6) we obtain $k=1$. 
Case 2. $\gamma(1) \in \operatorname{Sp}(2)_{+}^{0}$. In this case we have $\nu_{1}=\nu_{k}=1$. By computations (cf. [Lo1, Lo2]), we obtain

$$
\begin{aligned}
& \mu^{+}=1, \quad t^{+}=0, \quad i_{1}^{+}=i_{1}+\nu_{1}=i_{1}+1, \\
& \mu^{-}=0, \quad i_{1}^{-}=i_{1} .
\end{aligned}
$$

Thus by $1^{\circ}$ of Theorem 8.3 ,

$$
k\left(i_{1}^{-}-\mu^{-}\right)+\mu^{-} \geq i_{k} \geq k\left(i_{1}^{+}-\mu^{+}\right)+2 t^{+}+\mu^{+}-\nu_{k} .
$$

Together with (11.7) and (11.8) we obtain

$$
i_{k}=k i_{1} .
$$

By the conditions (M3) and $\nu_{k}=1$ we obtain

$$
1 \leq i_{k}=k i_{1} \leq 2 .
$$

Thus we must have $i_{1} \geq 1$ and $k \leq 2$. If $i_{1}=1$, then (11.7) yields $\mu^{+}=1$ and $i_{1}^{+}=2$. This contradicts Theorem 4.1. Therefore $i_{1} \geq 2$. By (11.11) we obtain $k=1$.

Case 3. $\gamma(1) \in \operatorname{Sp}(2)_{-}^{0}$. In this case, we have $\nu_{1}=\nu_{k}=1$. By computations (cf. [Lo1, Lo2]) we obtain

$$
\begin{aligned}
& \mu^{+}=0, \quad i_{1}^{+}=i_{1}+\nu_{1}=i_{1}+1, \\
& \mu^{-}=1, \quad t^{-}=k-1, \quad i_{1}^{-}=i_{1} .
\end{aligned}
$$

Thus, by $1^{\circ}$ of Theorem 8.3 ,

$$
k\left(i_{1}^{-}-\mu^{-}\right)+2 t^{-}+\mu^{-} \geq i_{k} \geq k\left(i_{1}^{+}-\mu^{+}\right)+\mu^{+}-\nu_{k} .
$$

Together with (11.12) and (11.13) we obtain

$$
i_{k}=k\left(i_{1}+1\right)-1 .
$$

By the conditions (M3) and $\nu_{k}=1$ we obtain

$$
1 \leq i_{k}=k\left(i_{1}+1\right)-1 \leq 2 .
$$

Then

$$
2 \leq k\left(i_{1}+1\right) \leq 3 .
$$

Thus we must have $i_{1} \geq 0$ and $k \leq 3$. If $i_{1}=0$, then (11.13) yields $\mu^{-}=1$ and $i_{1}^{-}=0$. This contradicts Theorem 4.1. Therefore $i_{1} \geq 1$. By (11.16) we obtain $k=1$.

The proof is complete.

Next we apply Theorem 11.1 to the autonomous asymptotically linear Hamiltonian systems defined on $\mathbf{R}^{2}$,

$$
\dot{x}=J H^{\prime}(x), \quad x \in \mathbf{R}^{2} .
$$

Theorem 11.2. Suppose the Hamiltonian function $H$ satisfies condition (H1). For $T>0$, let $x \in C^{2}\left(S_{T}, \mathbf{R}^{2}\right)$ be a nonconstant $T$-periodic solution of the system (11.17). Let the Maslov-type index of $x$ satisfy the following condition:

(X3) $2-\nu_{T}(x) \leq i_{T}(x) \leq 2$.

Then the solution $x$ possesses minimal period $T$. 
Proof. Let $\tau=T / k$ be the minimal period of $x$ for some $k \in \mathbf{N}$. Then (X3) implies that $2-\nu_{k \tau}(x) \leq i_{k \tau}(x) \leq 2$. Since $x$ is a nonconstant $\tau$-periodic solution of the autonomous system (11.16), we obtain $\nu_{\tau}(x) \geq 1$. Thus Theorem 11.1 yields $k=1$.

To continue the discussion, we need the following definition and the saddle point theorem.

Definition 11.3 (Definition 3.4 of [Gh]). Let $E$ be a $C^{2}$-Riemannian manifold, $B$ a closed subset of $E$. A family $\mathcal{F}(\alpha)$ is said to be a homological family of dimension $q$ with boundary $B$ if for some nontrivial class $\alpha \in H_{q}(E, B)$ the family $\mathcal{F}(\alpha)$ is defined by

$$
\mathcal{F}(\alpha)=\left\{A \subset E \mid \alpha \text { is in the image of } i_{*}: H_{q}(A, B) \rightarrow H_{q}(E, B)\right\},
$$

where $i_{*}$ is the homomorphism induced by the immersion $i: A \rightarrow E$.

Theorem 11.4 (Corollary 3.13 of [Gh]). As in Definition 11.3, for given $E, B$ and $\alpha$, let $\mathcal{F}(\alpha)$ be a homological family of dimension $q$ with boundary B. Suppose that $f \in C^{2}(E, \mathbf{R})$ satisfies condition (PS). Define

$$
c \equiv c(f, \mathcal{F}(\alpha))=\inf _{A \in \mathcal{F}(\alpha)} \sup _{x \in A} f(x) .
$$

Suppose that $\sup _{x \in B} f(x)<c$ and $f^{\prime}$ is Fredholm on

$$
\mathcal{K}_{c}=\left\{x \in E \mid f^{\prime}(x)=0, f(x)=c\right\} .
$$

Then there exists $x \in \mathcal{K}_{c}$ such that the Morse indices $m^{-}(x)$ and $m^{0}(x)$ of the functional $f$ at $x$ satisfy

$$
q-m^{0}(x) \leq m^{-}(x) \leq q .
$$

Now we apply Theorems 11.2 and 11.4 to the nonlinear system (11.17).

Theorem 11.5. Suppose that for $T>0$ the Hamiltonian function $H$ satisfies the conditions (H1)-(H4), (HT3), and (HT4). Then the system (11.17) possesses a periodic solution $x$ with minimal period $T$.

Proof. Since the proof is very similar to that of Theorem 10.1, we are very sketchy here. As in the proof of Theorem 10.1, we define a truncation function $H_{k}$ of $H$ so that $\left\|H_{k}^{\prime \prime}\right\|_{C}<+\infty$, and apply the saddle point reduction method to the problem. Let $E=Z$ with $2 d=\operatorname{dim} Z$, as in the proof of Theorem 10.1. Let $B=\partial Q$ and $\alpha=[Q] \in H_{d+2}(Z, B)$, where $Q$ is the closed cube defined by (10.23). Then $\alpha$ is nontrivial, and $\mathcal{F}(\alpha)$ defined by (11.18) is a homological family of dimension $d+2$ with boundary $B$. As we proved in Theorem 10.1, the functional $a$ defined by (10.18) satisfies the (PS) condition. It is well known that $a^{\prime}$ is Fredholm on $\mathcal{K}_{c}$ defined by (11.20) and (11.19). By Steps 4 and 5 of the proof of Theorem 10.1, we obtain

$$
\sup _{z \in B} a(z)<0<c(a, \mathcal{F}(\alpha)) .
$$

Thus by Theorem 11.4, we obtain a critical point $z \in Z$ of the functional $a$, and $x=u(z)$ is a nonconstant $T$-periodic solution of (10.17) which satisfies

$$
d+2-m^{0}(x) \leq m^{-}(x) \leq d+2,
$$


where $m^{-}(x)$ and $m^{0}(x)$ are the Morse index and the nullity of $a$ at its critical point $z$. So by Theorem 2.7 we obtain

$$
2-\nu_{T}(x) \leq i_{T}(x) \leq 2 .
$$

Now by (11.22) we can apply Theorem 11.2 to conclude that $x$ possesses minimal period $T$. As in the proof of Theorem 10.1, whenever $k$ is large enough, near the orbit of $x$ we have $H_{k}=H$. Thus $x$ is a nonconstant periodic solution of (11.17) with minimal period $T$. The proof is complete.

It is natural to ask whether Theorem 11.1 can be generalized to higherdimensional cases. Unfortunately, the answer is negative in general, because of the following example.

Example 11.6. For any integers $n \geq 2$ and $k \geq 2$ we choose $\theta \in(0,2 \pi)$ such that $k \theta=2 \pi+\chi \pi$ for some $\chi \in(0,2)$. Let $m=[n / 2]$. Using notation defined in the earlier sections, we define a path $\gamma:[0,1] \rightarrow \mathrm{Sp}(2 n)$ by

$$
\gamma(t)=F_{1}(t) \diamond \cdots \diamond F_{m}(t) \diamond Q_{1}(t) \diamond \cdots \diamond Q_{m}(t) \diamond D\left(2^{-t}\right) \diamond \cdots \diamond D\left(2^{-t}\right)
$$

where $F_{i}(t)=R(2 \pi t)$ and $Q_{i}(t)=R((\theta-2 \pi) t)$ for $1 \leq i \leq m$, and $D\left(2^{-t}\right)$ is defined by (3.2). Let $B(t)=-J \dot{\gamma}(t) \gamma^{-1}(t)$ for $0 \leq t \leq 1$. Then

$$
B \equiv B(t) \equiv A_{1} \diamond \cdots \diamond A_{m} \diamond B_{1} \diamond \cdots \diamond B_{m} \diamond D_{-}(\log 2) \diamond \cdots \diamond D_{-}(\log 2),
$$

where $A_{i}=2 \pi I$ and $B_{i}=(\theta-2 \pi) I$ for $1 \leq i \leq m$, and $D_{-}(\log 2)$ is defined by (4.11). Note that $\gamma(t)$ is the fundamental solution of the linear system

$$
\dot{x}=J B x, \quad x \in \mathbf{R}^{2 n} .
$$

By direct computations (cf. [Lo1, Lo2]) we obtain

$$
\begin{gathered}
i_{1}(\gamma)=m(2-1)+m(1-2)=0, \quad \nu_{1}(\gamma)=2 m \geq 2, \\
i_{k}(\tilde{\gamma})=m(2 k-1)+m(2+1-2 k)=2 m, \quad \nu_{k}(\tilde{\gamma})=2 m .
\end{gathered}
$$

Thus

$$
n+1-\nu_{k}(\tilde{\gamma}) \leq i_{k}(\tilde{\gamma}) \leq n+1 \quad \text { and } \quad \nu_{1}(\gamma) \geq 1 .
$$

Therefore when $n \geq 2$, the condition (11.27) does not yield any bound on $k$ in terms of $n$ in general.

\section{ACKNOWLEDGEMENT}

The senior author would like to thank the International Centre for Theoretical Physics, at Trieste, for support and hospitality during his visit in October and November of 1994. An earlier version of this paper appeared in its preprint series.

\section{REFERENCES}

[AZ] H. Amann and E. Zehnder, Nontrivial solutions for a class of non-resonance problems and applications to nonlinear differential equations, Ann. Scuola Norm. Sup. Pisa Cl. Sci. (4) 7 (1980), 539-603. MR 82b:47077

[AC] A. Ambrosetti and V. Coti Zelati, Solutions with minimal period for Hamiltonian systems in a potential well, Ann. Inst. H. Poincaré, Anal. Non Linéaire 4 (1987), 275-296. MR 88i: 58028

[AM] A. Ambrosetti and G. Mancini, Solutions of minimal period for a class of convex Hamiltonian systems, Math. Ann. 255 (1981), 405-421. MR 82j:58043

[Bo] R. Bott, On the iteration of closed geodesics and the Sturm intersection theory, Comm. Pure Appl. Math. 9 (1956), 171-206. MR 19:859f 
[Ch1] K. C. Chang, Solutions of asymptotically linear operator equations via Morse theory, Comm. Pure Appl. Math. 34 (1981), 693-712. MR 82m:58015

[Ch2] - Infinite dimensional Morse theory and multiple solution problems, Birkhäuser, Boston, 1993. MR 94e:58023

[CE1] F. Clarke and I. Ekeland, Hamiltonian trajectories having prescribed minimal period, Comm. Pure Appl. Math. 33 (1980), 103-116. MR 81e:70017

[CE2] - Nonlinear oscillations and boundary value problems for Hamiltonian systems, Arch. Rational Mech. Anal. 78 (1982), 315-333. MR 83h:58038

[CZ] C. Conley and E. Zehnder, Morse-type index theory for flows and periodic solutions for Hamiltonian equations, Comm. Pure Appl. Math. 37 (1984), 207-253. MR 86b:58021

[CD] R. Cushman and J. J. Duistermaat, The behavior of the index of a periodic linear Hamiltonian system under iteration, Adv. Math. 23 (1977), 1-21. MR 55:11296

[De] S. Deng, Minimal periodic solutions for a class of Hamiltonian equations, Acta Math. Sinica 27 (1984), 664-675. (Chinese) MR 87c:58038

[Ek1] I. Ekeland, Une théorie de Morse pour les systèmes hamiltoniens convexes, Ann. Inst. H. Poincaré Anal. Non Linéaire 1 (1984), 19-78. MR 85f:58023

[Ek2] _ An index theory for periodic solutions of convex Hamiltonian systems, Proc. Sympos. Pure Math. 45 (1986), 395-423. MR 87j:58023

[Ek3] , Convexity methods in Hamiltonian mechanics, Springer-Verlag, Berlin, 1990. MR 91f: 58027

[EH] I. Ekeland and H. Hofer, Periodic solutions with prescribed period for convex autonomous Hamiltonian systems, Invent. Math. 81 (1985), 155-188. MR 87b:58028

[GL] I. M. Gel'fand and V. B. Lidskiı̌, On the structure of the regions of stability of linear canonical systems of differential equations with periodic coefficients, Uspekhi Mat. Nauk 10 (1955), no. 1, 3-40; English transl., Amer. Math. Soc. Transl. (2) 8 (1958), 143-181. MR 17:482

[Gh] N. Ghoussoub, Location, multiplicity and Morse indices of min-max critical points, J.Reine Angew Math. 417 (1991), 27-76. MR 92e:58040

[GM1] M. Girardi and M. Matzeu, Some results on solutions of minimal period to superquadratic Hamiltonian equations, Nonlinear Anal. 7 (1983), 475-482. MR 84g:58092

[GM2] Solutions of minimal period for a class of nonconvex Hamiltonian systems and applications to the fixed energy problem, Nonlinear Anal. 10 (1986), 371-382. MR 87j:58038

[GM3] _ Periodic solutions of convex autonomous Hamiltonian systems with a quadratic growth at the origin and superquadratic at infinity, Ann. Mat. Pura Appl. (4) 147 (1987), 21-72. MR 89b:58074

[GM4] _ Dual Morse index estimates for periodic solutions of Hamiltonian systems in some nonconvex superquadratic case, Nonlinear Anal. 17 (1991), 481-497. MR 92k:58224

[GM5] _ Essential critical points of linking type and solutions of minimal period to superquadratic Hamiltonian systems, Nonlinear Anal. 19 (1992), 237-247. MR 93f:58030

[K1] W. Klingenberg, Lectures on closed geodesics, Springer-Verlag, Berlin, 1978. MR 57:17563

[LS] A. Lazer and S. Solimini, Nontrivial solutions of operator equations and Morse indices of critical points of min-max type, Nonlinear Anal. 12 (1988), 761-775. MR 89i:58018

[Lo1] Y. Long, Maslov-type index, degenerate critical points, and asymptotically linear Hamiltonian systems, Sci. China Ser. A 33 (1990), 1409-1419. MR 92d:58171

[Lo2] _ The structure of the singular symplectic matrix set, Sci. China Ser. A 34 (1991), 897-907. MR 93e:58065

[Lo3] _ Maslov-type index theory and asymptotically linear Hamiltonian systems, Dynamical Systems and Related Topics (Nagoya, 1990, K. Shiraiwa, ed.), World Sci. Publ., Singapore, 1991, pp. 333-341. MR 93f:58202

[Lo4] _ Estimates on the minimal period for periodic solutions of autonomous superquadratic second order Hamiltonian systems, In Nonlinear Analysis and Microlocal Analysis (K. C. Chang et al., eds.), World Sci. Publ., Singapore, 1992, pp. 168-175.

[Lo5] - The minimal period problem of periodic solutions for autonomous superquadratic second order Hamiltonian systems, J. Differential Equations 111 (1994), 147-174. MR 95k:58029

[Lo6] _ The minimal period problem of classical Hamiltonian systems with even potentials, Ann. Inst. H. Poincaré Anal. Non Linéaire 10 (1993), 605-626. MR 94m:58190 
[Lo7] - The index theory of Hamiltonian systems with applications, Science Press, Beijing, 1993. (Chinese)

[Lo8] - Nonlinear oscillations for classical Hamiltonian systems with bi-even subquadratic potentials, Nonlinear Anal. 24 (1995), 1665-1671. MR 96h:34079

[Lo9] Y. Long, A Maslov-type index theory for symplectic paths, Nankai Inst. of Math., Nankai Univ. Preprint, 1977.

[LD] Y. Long and D. Dong, Normal forms of symplectic matrices, Preprint, Nankai Inst. Math., Nankai Univ., 1995.

[LZ] Y. Long and E. Zehnder, Morse theory for forced oscillations of asymptotically linear Hamiltonian systems, Stochastic Processes Physics and Geometry (S. Albeverio et al., eds.), World Sci. Publ., Singapore, 1990, pp. 528-563. MR 92j:58019

[Mo] J. K. Moser, New aspects in the theory of stability of Hamiltonian systems, Comm. Pure Appl. Math. 11 (1958), 81-114. MR 20:3354

[Ra1] P. Rabinowitz, Periodic solutions of Hamiltonian systems, Comm. Pure Appl. Math. 31 (1978), 157-184. MR 57:7674

[Ra2] Minimax methods in critical point theory with applications to differential equations, CBMS Regional Conf. Ser. Math., no. 65, Amer. Math. Soc., Providence, RI, 1986. MR 87j:58024

[RS] J. Robbin and D. Salamon, The Maslov index for paths, Topology 32 (1993), 827-844. MR 94i: 58071

[So] S. Solimini, Morse index estimates in min-max theorems, Manuscripta Math. 63 (1989), 421-453. MR 90f:58028

[YS] V. A. Yakubovich and V. M. Starzhinskiu, Linear differential equations with periodic coefficients, Wiley, New York, 1975. MR 51:994

[Zh] S. Zhang, Doctoral Thesis, Nankai University, 1991.

Nankai Institute of Mathematics, Nankai University, Tianjin 300071, People's RePUBLIC OF CHINA

E-mail address: longym@sun.nankai.edu.cn

Current address, D. Dong: Department of Mathematics, State University of New York at Stony Brook, Stony Brook, New York 11794-3651

E-mail address: ddong@math.sunysb.edu 